\title{
MULTIPLICITY AND OPTICAL EXCESS ACROSS THE SUBSTELLAR BOUNDARY IN TAURUS
}

\author{
Adam L. Kraus, Russel J. White, and Lynne A. Hillenbrand \\ Department of Astrophysics, California Institute of Technology, MC 105-24, Pasadena, CA 91125; \\ alk@astro.caltech.edu,rjw@astro.caltech.edu, lah@astro.caltech.edu \\ Received 2005 November 18; accepted 2006 February 18
}

\begin{abstract}
We present the results of a high-resolution imaging survey of 22 brown dwarfs and very low mass stars in the nearby ( $\sim 145 \mathrm{pc})$ young $(\sim 1-2 \mathrm{Myr})$ low-density star-forming region Taurus-Auriga. We obtained images with the Advanced Camera for Surveys High Resolution Channel on HST through the F555W $(V)$, F775W ( $\left.i^{\prime}\right)$, and F850LP $\left(z^{\prime}\right)$ filters. This survey confirmed the binarity of [BHS98] MHO 8 (hereafter MHO-Tau-8) and discovered a new candidate binary system, [SS94] V410 X-ray 3 (hereafter V410-Xray3), resulting in a binary fraction of $9_{-3}^{+10 \%}$ at separations $>4$ AU. Both binary systems are tight $(<10 \mathrm{AU})$, and they possess mass ratios of 0.75 and 0.46 , respectively. The binary frequency and separations are consistent with low-mass binary properties in the field, but the mass ratio of V410-Xray3 is among the lowest known. We find that the binary frequency is higher for very low mass stars and high-mass brown dwarfs than for lower mass brown dwarfs, implying either a decline in frequency or a shift to smaller separations for the lowest mass binaries. Combining these results with multiplicity statistics for higher mass Taurus members suggests a gradual decline in binary frequency and separation toward low masses. The implication is that the distinct binary properties of very low-mass systems are set during formation and that the formation process is similar to the process that creates higher mass stellar binaries, but occurs on a smaller scale. We combine the survey detection limits with models for planetary-mass objects to show that there are no planets or very low-mass brown dwarfs with mass $>3 M_{\mathrm{J}}$ at projected separation $>40$ AU orbiting any of the Taurus members in our sample, implying that planetary-mass companions at wide separations are rare. Finally, based on fits to the optical and near-infrared spectral energy distributions, we identify several BDs with significant ( $\gtrsim 1 \mathrm{mag}) V$-band excesses. The excesses appear to be correlated with signatures of accretion and if attributed to accretion luminosity may imply mass accretion rates several orders of magnitude above those inferred from line profile analyses.
\end{abstract}

Subject headings: binaries: visual — stars: low-mass, brown dwarfs — stars: pre-main-sequence

\section{INTRODUCTION}

Brown dwarfs (BDs) are objects with masses between those of stars and planets, insufficient to maintain stable fusion reactions in their cores. BDs comprise a significant fraction of the total (sub)stellar content of the galaxy and are among our nearest neighbors (Reid et al. 2004); in the past decade, field surveys have discovered several hundreds of BDs in the solar neighborhood (e.g., Delfosse et al. 1997; Kirkpatrick et al. 1999; Leggett et al. 2000). Soon after BDs were discovered, it was found that many, like stars, are members of binary systems. However, the properties of binary systems near and below the substellar boundary $\left(M_{\text {prim }}<0.2 M_{\odot}\right)$ appear to be fundamentally different from those of higher mass stars $\left(0.3<M_{\text {prim }}<1.0 M_{\odot}\right)$. Multiplicity surveys of field $\mathrm{T}$ dwarfs (Burgasser et al. 2003), L dwarfs (Koerner et al. 1999; Close et al. 2003; Bouy et al. 2003; Gizis et al. 2000), and late M dwarfs (Siegler et al. 2005) have found lower binary frequencies $(\sim 15 \%$ vs. $40 \%-55 \%)$ and smaller binary separations $(<20$ vs. $<1000 \mathrm{AU})$ than for field stars (Duquennoy \& Mayor 1991; Fischer \& Marcy 1992; Halbwachs et al. 2003).

These results demonstrate that field binary properties depend on mass. Unfortunately, binary frequencies for field stars are only reported for broad mass ranges, so they do not place strong constraints on the functional form of this dependence. Various groups interpret the transition in binary properties as either a sharp break near the stellar/substellar boundary (Kroupa et al. 2003; Close et al. 2003) or a smooth mass dependence (Luhman 2004b). Also, since field BD systems are typically old and possess lower binding energies than stellar binaries of equal separation, the re- sults could be biased by the dynamical disruption of wide, lowmass systems. To begin testing this possibility, we performed a small multiplicity survey of low-mass stars and BDs in the nearby young OB association Upper Scorpius (Kraus et al. 2005) and T association Taurus (this work). In Upper Sco, we found several young binary systems, but the binary frequency, separations, and mass ratios were consistent with the field and somewhat $(\sim 2 \sigma)$ inconsistent with higher mass members of Upper Sco (Kohler et al. 2000). This suggests that dynamical evolution after the $\mathrm{T}$ Tauri stage probably does not produce the unique binary parameters of BDs; instead, the implication is that the mechanism by which binaries form depends on mass.

Recent efforts to model low-mass binary formation have typically assumed that a cluster of 5-10 protostellar embryos form from a single fragmenting cloud core (e.g., Kroupa 1995; Sterzik \& Durisen 1998; Kroupa \& Bouvier 2003; Kroupa et al. 2003; Delgado-Donate et al. 2003; Hubber \& Whitworth 2005); these embryos would then undergo dynamical evolution to form single stars and stable multiple systems. However, the frequency of multiple stellar systems $\left(M_{\text {prim }}>0.3 M_{\odot}\right)$ in the field (35\%57\%; Duquennoy \& Mayor 1991; Reid \& Gizis 1997) and in young associations $(50 \%-80 \%$; Kohler et al. 2000 ; R. J. White et al. 2006, in preparation) has been interpreted by Goodwin \& Kroupa (2005) to mean that the collapse and fragmentation of a cloud core produces only two or three stars. Larger systems would eject more single stars and tight binaries than are observed. Reipurth \& Clarke (2001) have suggested that evolution to a dynamically stable state could occur early in the formation process; the ejected embryos would then cease accretion and become BDs. 
The ejection process would preferentially disrupt wide BD binary systems, causing the deficit of wide systems seen in lowmass field binaries. However, simulations by Bate et al. (2003) find that the corresponding binary frequencies and separations $(<5 \%$ and $<10 \mathrm{AU})$ are too low to be consistent with the field. Also, some wide very low mass binaries have recently been discovered in the field (Gizis et al. 2000; Phan-Bao et al. 2005; Billeres et al. 2005). These systems are very weakly bound and most likely would not survive the ejection process, although recent simulations by Bate \& Bonnell (2005) suggest that they could form via simultaneous ejection of previously unbound objects. Finally, some models predict that ejection would alter other properties of BDs (spatial and velocity dispersion, disk lifetime, and accretion frequency). The preponderance of observations show that these properties are similar in the stellar and substellar regimes (White \& Basri 2003; Luhman 2004b; White \& Hillenbrand 2004; Mohanty et al. 2005); the strong similarity between the two regimes suggests that brown dwarfs form in a manner similar to stars and thus that BD binaries form like stellar binaries, although possibly on a smaller scale. The implication is that all binaries share a common formation mechanism, fragmentation of a single collapsing cloud core, and that this mechanism features a mass dependence that remains unexplained by theoretical models.

Multiplicity surveys of the field and of nearby stellar populations (e.g., Kohler et al. 2000; Luhman et al. 2005a; R. J. White et al. 2006, in preparation) have placed some constraints on the form of this mass dependence. In particular, R. J. White et al. (2006, in preparation) studied the mass dependence of multiplicity in a speckle interferometry survey of the nearby $(\sim 145 \mathrm{pc})$ young $(\sim 1-2 \mathrm{Myr}) \mathrm{T}$ association Taurus-Auriga. This survey included objects from $1.5 M_{\odot}$ to the substellar boundary and found that the separation distribution and mass ratio distribution functions were mass dependent. Their results for the binary frequency were inconclusive, but suggested a possible slow decline with mass. Better statistics will be required for very low mass binaries in order to confirm this trend. In this paper, we present the results of an complementary imaging multiplicity survey of very low mass stars and brown dwarfs to near the planetary-mass regime in Taurus-Auriga.

\section{OBSERVATIONS AND DATA REDUCTION}

\subsection{Sample Selection}

The Taurus-Auriga association has been the target of many recent wide-field surveys to detect new low-mass members (e.g., Briceno et al. 1998, 2002; Martin et al. 2001; Luhman et al. 2003a). These surveys identified candidate members based on their location on an optical or near-infrared color-magnitude diagram, and membership was then confirmed spectroscopically via the detection of lithium absorption, excess $\mathrm{H} \alpha$ emission, or low surface gravity, all of which are indicators of youth. We selected all 18 confirmed Taurus members with spectral type later than M5.5 discovered by these surveys. Three additional targets ([SS94] V410 X-ray 3, hereafter V410-Xray-3; [SS94] V410 Anon 13, hereafter V410-Anon-13; and GM Tau) are previously known Taurus members, which have also been confirmed to possess spectral types in this range (Strom \& Strom 1994; White \& Basri 2003). Our final target (LH 0419+15) was chosen from a survey for Hyades members by Reid \& Hawley; it is the only member of their survey with a spectroscopic detection of lithium, and they classify it as a likely Taurus member with spectral type M7 based on its apparent youth and distance. We list these 22 targets and their discovery or confirmation references in Table 1; this was a complete list of known Taurus members with spectral types
TABLE 1

OBSERVATIONS

\begin{tabular}{|c|c|c|c|c|c|}
\hline \multirow[b]{2}{*}{ TARGET } & \multirow[b]{2}{*}{ DAtE $^{a}$} & \multicolumn{3}{|c|}{ Exposure Times (s) } & \multirow[b]{2}{*}{ Discovery } \\
\hline & & F555W & F775W & F850LP & \\
\hline CFHT-Tau-1 ........ & 2904.3 & 510 & 300 & 200 & Martin et al. (2001) \\
\hline CFHT-Tau-2 . & 2904.3 & 510 & 300 & 200 & Martin et al. (2001) \\
\hline CFHT-Tau-3 ....... & 2901.5 & 510 & 300 & 200 & Martin et al. (2001) \\
\hline CFHT-Tau- 4 ........ & 2857.5 & 510 & 300 & 200 & Martin et al. (2001) \\
\hline KPNO-Tau-1 ....... & 2881.2 & 510 & 300 & 200 & Briceno et al. (2002) \\
\hline KPNO-Tau-2 ....... & 2903.3 & 510 & 300 & 200 & Briceno et al. (2002) \\
\hline KPNO-Tau-3 ...... & 2901.4 & 510 & 300 & 200 & Briceno et al. (2002) \\
\hline KPNO-Tau-4 ...... & 2904.4 & 510 & 300 & 200 & Briceno et al. (2002) \\
\hline KPNO-Tau-5 ....... & 2884.2 & 350 & $300 / 30$ & $200 / 20$ & Briceno et al. (2002) \\
\hline KPNO-Tau- 6 ...... & 3054.5 & 510 & 300 & 200 & Briceno et al. (2002) \\
\hline KPNO-Tau-7 ...... & 2901.6 & 510 & 300 & 200 & Briceno et al. (2002) \\
\hline KPNO-Tau-8 ...... & 2850.5 & 350 & $300 / 30$ & $200 / 20$ & Briceno et al. (2002) \\
\hline KPNO-Tau-9 ...... & 3025.8 & 510 & 300 & 200 & Briceno et al. (2002) \\
\hline KPNO-Tau-12. & 3028.7 & 510 & 300 & 200 & Luhman et al. (2003a) \\
\hline KPNO-Tau-14.. & 2897.5 & 350 & $300 / 30$ & $200 / 20$ & Luhman et al. (2003a) \\
\hline MHO-Tau- $4 \ldots .$. & 2893.5 & 350 & $300 / 30$ & $200 / 20$ & Briceno et al. (1998) \\
\hline MHO-Tau-5........ & 3023.0 & 350 & $300 / 30$ & $200 / 20$ & Briceno et al. (1998) \\
\hline MHO-Tau- $8 \ldots \ldots \ldots$ & 3028.5 & 350 & $300 / 30$ & $200 / 20$ & Briceno et al. (1998) \\
\hline LH $0419+15 \ldots \ldots$ & 2901.0 & 510 & 300 & 200 & Reid \& Hawley (1999) \\
\hline V410-Xray3 ..... & 3029.4 & 350 & $300 / 30$ & $200 / 20$ & Strom \& Strom (1994) \\
\hline V410 Anon13.. & 2903.4 & 510 & 300 & 200 & Strom \& Strom (1994) \\
\hline GM Tau..... & 2850.6 & 350 & $300 / 30$ & $200 / 20$ & White \& Basri (2003) \\
\hline
\end{tabular}

a Observation date: JD-2,450,000.

later than M5.5 at the time the observations were proposed (2003 January). Since our targets include both very low mass stars and brown dwarfs, we hereafter refer to them as very low mass objects, or VLMOs.

\subsection{Observations}

Our images were obtained with the Advanced Camera for Surveys High Resolution Camera on the Hubble Space Telescope $(H S T)$, which has a field of view of $26^{\prime \prime} \times 29^{\prime \prime}$ and distortioncorrected pixel size of 27 mas pixel $^{-1}$. In Table 2, we summarize the exposure time and epoch of observation for each target (program ID: 9853). Observations were made between 2003 September and 2004 January with the filters F555W $(V)$, F775W $\left(i^{\prime}\right)$, and F850LP $\left(z^{\prime}\right)$ at two dither positions near the center of the detector and with two exposures per position. Total integration times were 510,300 , and $200 \mathrm{~s}$, respectively. The F555W exposure times for the brightest objects were reduced to $350 \mathrm{~s}$ to allow for additional short exposures in F775W and F850LP, which were close to the saturation limit in the full-length exposures and were saturated in the case of MHO-Tau-5 and [BHS98] MHO 8 (hereafter MHOTau-8). Saturation was permitted in the full-length images to allow for comparable sensitivities to faint companions at wide separations. We chose the $V$ band to maximize angular resolution (diffraction limit $\theta_{\mathrm{res}, V}=58 \mathrm{mas}$ ) and the $i^{\prime}$ and $z^{\prime}$ bands to maximize sensitivity to very low mass companions.

The raw images were calibrated and distortion-corrected by the CALACS pipeline during on-the-fly reprocessing (Mack et al. 2003). Some cosmic rays remained, but their morphologies were substantially different from stellar point-spread functions (PSF), so they were easily identified by visual inspection.

\subsection{Data Reduction}

Potential point sources were identified with the IRAF task DAOPHOT/DAOFIND, and we measured aperture photometry 
TABLE 2

Photometry of Very Low Mass Objects in Taurus

\begin{tabular}{|c|c|c|c|c|c|c|}
\hline Name & $V^{\mathrm{a}}$ & $i^{\prime \mathrm{a}}$ & $z^{\prime \mathrm{a}}$ & $J^{\mathrm{b}}$ & $H^{\mathrm{b}}$ & $K^{\mathrm{b}}$ \\
\hline CFHT-Tau-2 …........... & $22.520 \pm 0.014$ & $18.112 \pm 0.002$ & $16.307 \pm 0.002$ & $13.754 \pm 0.021$ & $12.762 \pm 0.021$ & $12.169 \pm 0.017$ \\
\hline CFHT-Tau- $4 \ldots \ldots \ldots \ldots \ldots$ & $21.556 \pm 0.008$ & $16.920 \pm 0.001$ & $14.951 \pm 0.001$ & $12.168 \pm 0.020$ & $11.008 \pm 0.019$ & $10.332 \pm 0.016$ \\
\hline KPNO-Tau-1 .................. & $24.063 \pm 0.044$ & $19.572 \pm 0.004$ & $17.596 \pm 0.003$ & $15.101 \pm 0.038$ & $14.247 \pm 0.037$ & $13.772 \pm 0.034$ \\
\hline KPNO-Tau-2 ................. & $21.376 \pm 0.007$ & $17.670 \pm 0.002$ & $16.074 \pm 0.001$ & $13.925 \pm 0.022$ & $13.241 \pm 0.027$ & $12.753 \pm 0.020$ \\
\hline KPNO-Tau-5 ................. & $19.690 \pm 0.004$ & $16.226 \pm 0.001$ & $14.706 \pm 0.001$ & $12.640 \pm 0.020$ & $11.918 \pm 0.022$ & $11.536 \pm 0.016$ \\
\hline KPNO-Tau-6 ................... & $22.292 \pm 0.012$ & $19.097 \pm 0.003$ & $17.301 \pm 0.003$ & $14.995 \pm 0.028$ & $14.197 \pm 0.038$ & $13.689 \pm 0.036$ \\
\hline KPNO-Tau-7 ................... & $22.068 \pm 0.011$ & $18.358 \pm 0.002$ & $16.661 \pm 0.002$ & $14.521 \pm 0.030$ & $13.828 \pm 0.026$ & $13.272 \pm 0.032$ \\
\hline KPNO-Tau-8 .................. & $19.261 \pm 0.003$ & $16.147 \pm 0.001$ & $14.863 \pm 0.001$ & $12.946 \pm 0.018$ & $12.367 \pm 0.019$ & $11.985 \pm 0.020$ \\
\hline KPNO-Tau-9 ................. & $24.918 \pm 0.084$ & $20.035 \pm 0.006$ & $18.056 \pm 0.004$ & $15.497 \pm 0.042$ & $14.660 \pm 0.039$ & $14.185 \pm 0.053$ \\
\hline KPNO-Tau-12 ............... & $23.228 \pm 0.023$ & $20.781 \pm 0.009$ & $18.998 \pm 0.006$ & $16.305 \pm 0.085$ & $15.483 \pm 0.096$ & $14.927 \pm 0.092$ \\
\hline KPNO-Tau-14 ................ & $20.735 \pm 0.007$ & $16.297 \pm 0.001$ & $14.502 \pm 0.001$ & $11.907 \pm 0.019$ & $10.805 \pm 0.021$ & $10.269 \pm 0.018$ \\
\hline MHO-Tau-8 В ............... & $19.525 \pm 0.048$ & $15.611 \pm 0.060$ & $13.934 \pm 0.013$ & $\ldots$ & . & $\ldots$ \\
\hline LH $0419+15 \ldots \ldots \ldots \ldots \ldots$ & $21.835 \pm 0.010$ & $18.028 \pm 0.002$ & $16.488 \pm 0.002$ & $14.364 \pm 0.029$ & $13.549 \pm 0.027$ & $13.079 \pm 0.035$ \\
\hline 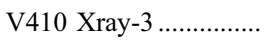 & $18.319 \pm 0.002$ & $15.046 \pm 0.001$ & $13.629 \pm 0.001$ & $11.544 \pm 0.018$ & $10.817 \pm 0.021$ & $10.446 \pm 0.017$ \\
\hline V410 Xray-3 A............... & $\ldots$ & $15.046 \pm 0.014$ & $13.812 \pm 0.011$ & $\ldots$ & $\ldots$ & $\ldots$ \\
\hline V410 Xray-3 B ............... & $\ldots$ & $20.032 \pm 0.446$ & $15.658 \pm 0.028$ & $\ldots$ & $\ldots$ & $\ldots$ \\
\hline V410 Anon-13 ............... & $22.175 \pm 0.012$ & $17.665 \pm 0.002$ & $15.782 \pm 0.001$ & $12.954 \pm 0.019$ & $11.659 \pm 0.020$ & $10.958 \pm 0.015$ \\
\hline GM Tau ............................ & $17.577 \pm 0.001$ & $15.169 \pm 0.001$ & $13.908 \pm 0.001$ & $12.804 \pm 0.019$ & $11.586 \pm 0.017$ & $10.632 \pm 0.016$ \\
\hline
\end{tabular}

a Uncertainties are statistical only; systematic uncertainties due to aperture corrections and conversion to standard systems are $\sim 0.03$ mag.

${ }^{\mathrm{b}}$ Near-infrared photometry is taken from the Two Micron All Sky Survey (Cutri et al. 2003). We quote total system magnitudes for the two binary systems.

and PSF-fitting photometry for all objects in each field using the DAOPHOT tasks PHOT and ALLSTAR. We describe these data reduction procedures in more detail in our companion paper on VLMO multiplicity in Upper Sco (Kraus et al. 2005), and the DAOPHOT package is described by Stetson (1987). We report aperture photometry for all isolated objects and PSF photometry for all close binaries. PSF magnitudes were corrected to match aperture magnitudes based on results for the 20 apparently isolated Taurus members in our sample $(\S 3.1)$. A preliminary PSF for each filter was constructed from the 20 well-sampled VLMOs that appeared isolated under visual inspection. Since all of the targets were located near the center of the chip and have similar temperatures, image distortion and target color should not be important. There was some variation in the PSF FWHM from target to target $( \pm 5 \%)$, which we attribute to small orbit-to-orbit changes in focus. We investigated this by dividing our sample into two groups, based on whether the target PSF appeared to be narrower or wider than the average PSF, and constructing new average PSFs for each group. We then reran PSF photometry, but the modest decrease in the residuals did not reveal any companions that were not previously identified. Since it is not possible to determine which PSF is appropriate for blended binaries, we proceed using only the average PSF for the entire group. This choice could lead to systematic errors in the calculation of binary properties; we discuss these errors in more detail in $\S 3.4$.

Transformations to ground-based magnitudes $\left(V, \operatorname{SDSS} i^{\prime}\right.$, and $\operatorname{SDSS} z^{\prime}$ ) were described in detail in Kraus et al. (2005). We found constant corrections that do not depend significantly on temperature or surface gravity: $m_{555}-m_{V}=-0.16 \pm 0.03, m_{775}-m_{i^{\prime}}=$ $+0.07 \pm 0.03$, and $m_{850}-m_{z^{\prime}}=+0.03 \pm 0.03$. The transformed magnitudes and uncertainties are listed in Table 2. The statistical uncertainties correspond to either the photon noise (for aperture photometry; typically $<0.01 \mathrm{mag}$ ) or the goodness of fit (for PSFfitting photometry; 0.01-0.06 mag for objects with significant fits). Systematic uncertainties in the magnitude transformations and aperture corrections are $\sim 0.03 \mathrm{mag}$. The photometry calculated from short exposures was consistent with that from long exposures, so we report only the long exposures.

\section{RESULTS}

\subsection{VLMO Binaries}

The present survey found no fully resolved low-mass binaries. However, as we discuss in Kraus et al. (2005), one limitation in ALLSTAR-based PSF photometry is that binaries with very close $(\lesssim \lambda / D)$ separations are often not identified, even when their combined PSF is elongated at a high confidence level. DAOFIND, the task that identifies potential objects in the images, identifies point sources based only on the presence of a distinct peak. Thus, automated photometry will be biased against the detection of very close binaries. This limitation can be overcome for known or suspected binaries by manually adding a second point source in approximately the correct location and letting ALLSTAR recenter it to optimize the fit. We have already used this method to recover photometry for the candidate binary system USco-109 in Kraus et al. (2005).

In Figures 1-3, we illustrate this technique with contour plots of the known VLMO binary MHO-Tau-8 (discovered by R. J. White et al. 2006, in preparation) and independently rediscovered here), the new candidate VLMO binary V410-Xray3, and the apparently single VLMO MHO-Tau-4 in the F555W, F775W, and F850LP filters. Since the long exposures in $i^{\prime}$ and $z^{\prime}$ for MHOTau- 8 were saturated, we show only the short exposures. Neither of the two candidate systems is obviously resolved, and neither 


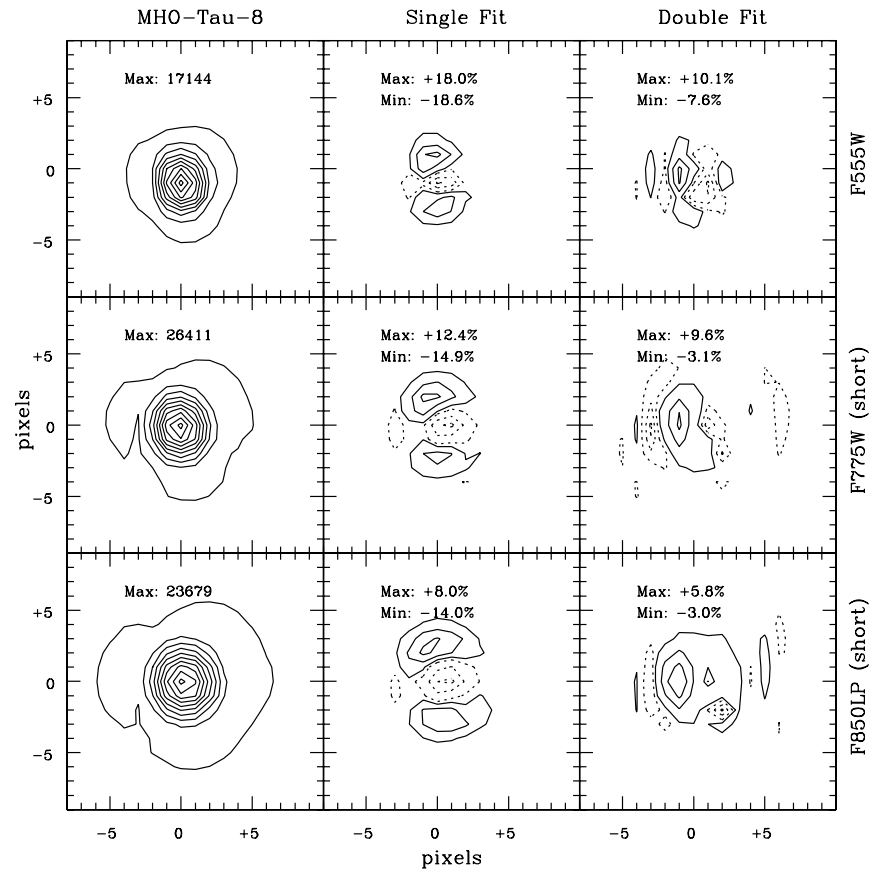

Fig. 1.-Contour plots of MHO-Tau-8 for all three filters. The first column shows MHO-Tau- 8 , the second column shows the residuals from fitting it with one source, and the last column shows the residuals from fitting it with two sources. For residuals, contours are drawn at the $90 \%, 50 \%$, and $10 \%$ levels of maximum (solid lines) and minimum (dashed lines). The peak pixel value in each original image is shown; the positive and negative peaks of the residuals are reported as a percentage of the original peak value.

was consistently reported as a double source by DAOFIND, but the PSF for MHO-Tau-8 is slightly elongated along the $y$-axis and that of V410-Xray 3 is slightly elongated along the $x$-axis relative to MHO-Tau-4.

In Figure 1, we present contour plots of MHO-Tau-8 in each filter. The first column shows the original image, the second column shows the result from fitting with one point source, and the last column shows the result from fitting with two point sources. The maximum and minimum pixel values are also given to allow quantitative comparison of the residuals to the original images. The common position angle of the residuals in the single-source fit seems to imply that this elongation is a real effect, and not simply noise. Normal levels of jitter were reported in the observation $\log$, so this is also unlikely to be a systematic effect. Unfortunately, there were no other bright objects in the field to serve as PSF references. However, as we summarize in Table 3, the system properties in each filter are consistent with previous observations and expected orbital motion ( $(5.3)$. Since MHO-Tau- 8 was independently identified as a binary system by R. J. White et al. (2006, in preparation), we regard it as a confirmed discovery.

In Figure 2, we present similar contour plots of V410-Xray3, showing both the long and short exposures in $i^{\prime}$ and $z^{\prime}$. The residuals from the single-source fit are roughly aligned, and the jitter levels were normal, as in the case of MHO-Tau-8, but the separations of the residuals are marginally lower, implying a smaller separation or larger flux ratio. ALLSTAR was unable to fit two point sources in the $V$ image and the fit for the secondary in the $i^{\prime}$ images was not statistically significant, but the fit for the $z^{\prime}$ images appears consistent and statistically significant in both the short and long exposures. The number statistics for the $i^{\prime}$ and $z^{\prime}$ images are similar, and the $z^{\prime}$ filter has lower resolution; therefore the superior fit for the $z^{\prime}$ images suggests that a possible companion may be much cooler and redder. We summarize the system

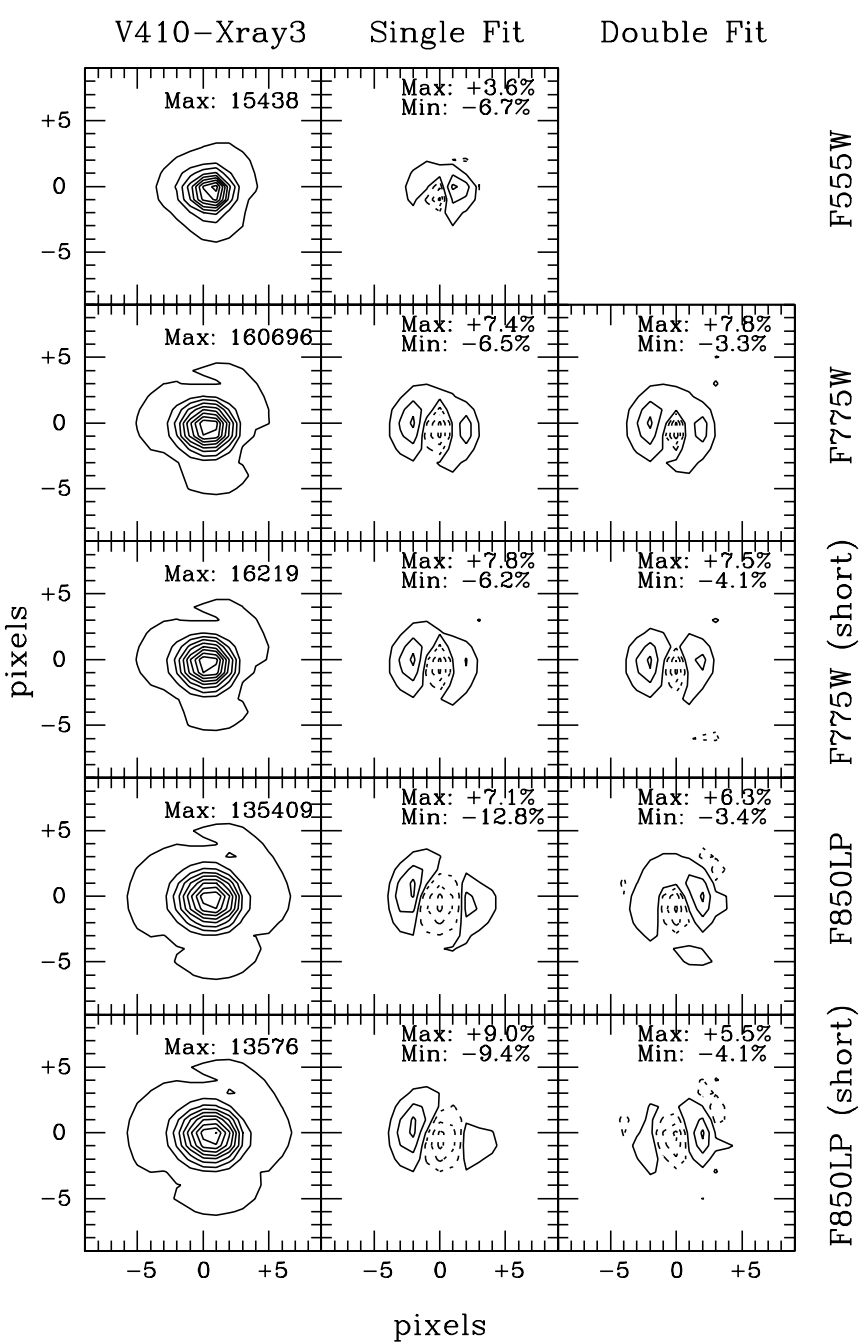

FIg. 2.-Contour plots of V410-Xray3 for all three filters. The first column shows V410-Xray3, the second column shows the residuals from fitting it with one source, and the last column shows the residuals from fitting it with two sources. For residuals, contours are drawn at the $90 \%, 50 \%$, and $10 \%$ levels of maximum (solid lines) and minimum (dashed lines). The peak pixel value in each original image is shown; the positive and negative peaks of the residuals are reported as a percentage of the original peak value.

parameters as calculated from each image in Table 3, although the results for the $i^{\prime}$ images should be used with caution.

The significant residuals in the double-source fit and the scatter in system properties suggests that even if V410-Xray3 is a binary, the measured parameters are not very reliable. Since there are no background stars for comparison and no other observations to support its multiplicity, we suggest its classification as a candidate binary. Follow-up observation to confirm its existence and properties should be a priority, since if confirmed, its small separation and corresponding short orbital period ( $\sim 50 \mathrm{yr}$ for a circular orbit) could allow for a dynamical mass determination in less than a decade. Our subsequent analysis will consider V410-Xray3 as a candidate binary, but since its binarity has not been confirmed or disproved, our discussion will reflect both possibilities. $^{1}$

In Figure 3, we illustrate the typical results for single stars with contour plots of MHO-Tau- 4 and the residuals after fitting with a

\footnotetext{
${ }^{1}$ Observations conducted in 2006 February with the Keck II telescope and Laser Guide Star Adaptive Optics have confirmed this candidate and will be reported in a future publication.
} 


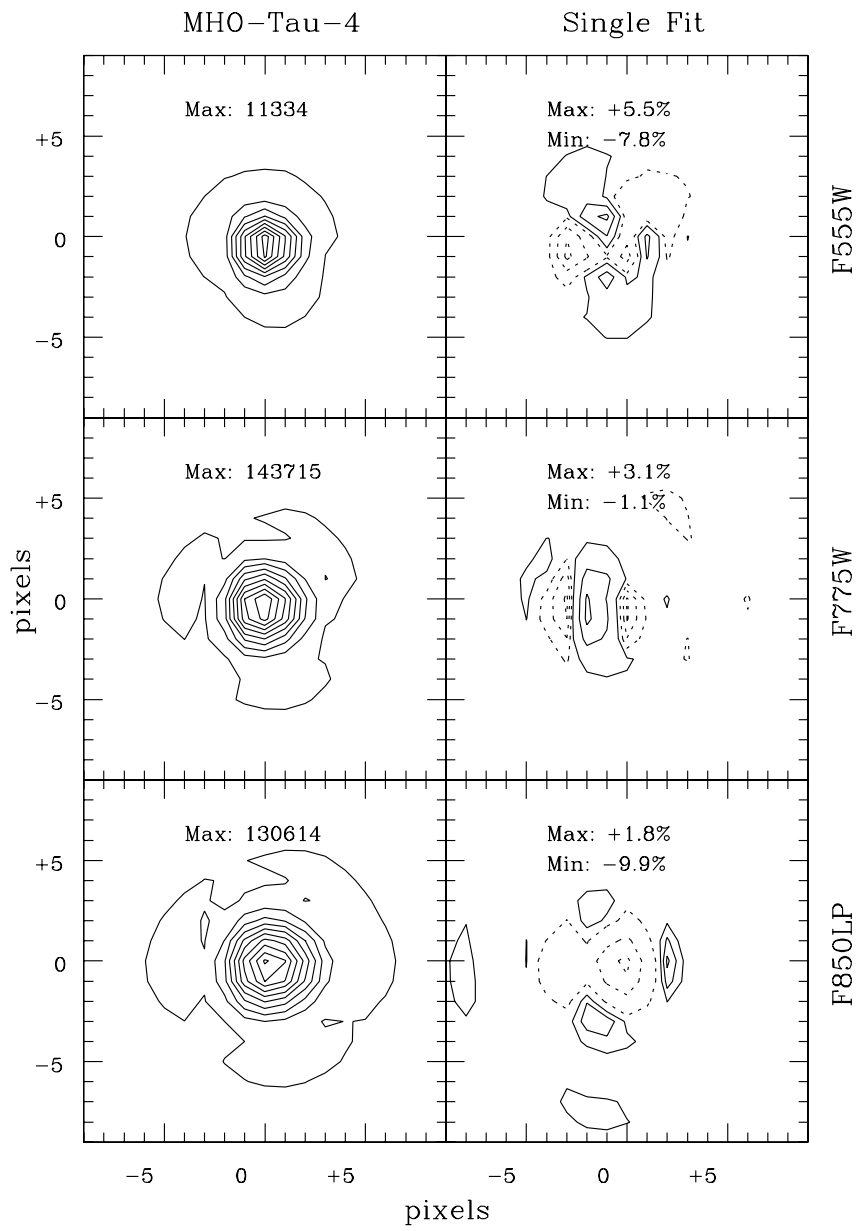

FIG. 3.-Contour plots of MHO-Tau-4 for all three filters. The first column shows MHO-Tau-4 and the second column shows the residuals from fitting it with one source. For residuals, contours are drawn at the $90 \%, 50 \%$, and $10 \%$ levels of maximum (solid lines) and minimum (dashed lines). The peak pixel value in each original image is shown; the positive and negative peaks of the residuals are reported as a percentage of the original peak value. single point source. We could not obtain a statistically significant fit for two point sources, suggesting that there are no binary companions at separations $\gtrsim 4 \mathrm{AU}$ with mass ratio near unity.

One target VLMO, KPNO-Tau-14, was reported as a possible double-lined spectroscopic binary by Mohanty et al. (2005). We did not detect any PSF elongation for this target. Since the components of SB2s have similar brightness, the detection limits we find in $\S 3.3$ imply its separation is less than our inner detection limit ( $\sim \mathrm{AU})$. Since it falls inside our survey limits, we will not consider it a binary in our discussion.

\subsection{VLMOs and Background Stars}

In Figure 4, we present an $i^{\prime}$ versus $i^{\prime}-z^{\prime}$ color-magnitude diagram of the 20 apparently single VLMO targets (filled circles), the components of the two candidate binary systems ( filled circles with error bars), and all other objects that were clearly resolved and detected at the $5 \sigma$ level in both filters (open circles). The candidate secondary component of V410-Xray3 is well off the right side of the graph; its extremely red color $\left(i^{\prime}-z^{\prime}=\right.$ $4.374)$ is probably due to the high uncertainty in its $i^{\prime}$ fit $(\S 3.1)$; since the secondary has an approximate spectral type of M7.7 (§ 4.3), we expect it to possess colors similar to those of CFHTTau-3, $i^{\prime}-z^{\prime}=2$, and thus $i^{\prime}=17.5$. Also shown are the average main sequence at the distance of Taurus (Hawley et al. 2002), 1 and 2 Myr isochrones based on the evolutionary models of Baraffe et al. (1998), and a reddening vector based on the extinction relations reported in Schlegel et al. (1998). The location of the isochrone is determined by converting the predicted $I_{\mathrm{C}}$ magnitudes to $i^{\prime}$ magnitudes using the color $\left(I_{\mathrm{C}}-i^{\prime}=0.40\right)$ derived as in $\S 2$ and by assuming the $i^{\prime}-z^{\prime}$ colors found from the SDSS field main sequence for older, more massive dwarfs by Hawley et al. (2002). As we show in $\S 4.3$, the assumption of dwarf $i^{\prime}-z^{\prime}$ colors for these targets appears to be valid. The DUSTY models (Chabrier et al. 2000) should not be required for these VLMOs due to their high temperatures at this young age, although we will use these models to determine limits on faint companions ( $§ 4.2)$.

In Figure 4, the binary components are located above the SDSS main sequence and well above the background population, so they

TABLE 3

Binary Properties

\begin{tabular}{|c|c|c|c|c|c|c|}
\hline Date $^{a}$ & $\begin{array}{l}\text { Separation } \\
\text { (mas) }\end{array}$ & $\begin{array}{l}\text { Position Angle } \\
\text { (deg) }\end{array}$ & Filter & $\begin{array}{c}\text { Flux Ratio } \Delta m \\
\text { (mag) }\end{array}$ & $\Delta \mathrm{SpT}$ & $q$ \\
\hline \multicolumn{7}{|c|}{ MHO-Tau-8 } \\
\hline $1143^{\mathrm{b}}$ & 37 & 311 & K & 0.02 & $\ldots$ & $\ldots$ \\
\hline 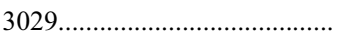 & 54 & 269 & $V$ & 1.28 & $\ldots$ & $\ldots$ \\
\hline 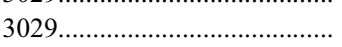 & 39 & 272 & $i^{\prime}$ & 0.70 & $\cdots$ & $\cdots$ \\
\hline 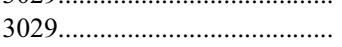 & 40 & 268 & $z^{\prime}$ & 0.378 & $\cdots$ & $\cdots$ \\
\hline$\langle 3029\rangle^{\mathrm{c}} \ldots \ldots \ldots \ldots \ldots \ldots$ & $44 \pm 8$ & $270 \pm 2$ & $\ldots$ & $\ldots$ & $0.6 \pm 0.1$ & $0.75 \pm 0.07$ \\
\hline \multicolumn{7}{|c|}{ V410-Xray3 } \\
\hline 3029 & 56 & 192 & $i^{\prime}$ (long) & 5.0 & $\ldots$ & $\ldots$ \\
\hline 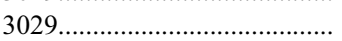 & 45 & 205 & $z^{\prime}$ & 1.85 & $\ldots$ & $\ldots$ \\
\hline 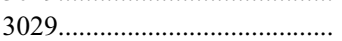 & 46 & 234 & $i^{\prime}$ (short) & 3.7 & $\ldots$ & $\ldots$ \\
\hline 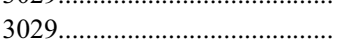 & 43 & 203 & $z^{\prime}$ & 1.55 & $\cdots$ & $\cdots$ \\
\hline$\langle 3029\rangle^{\mathrm{c}}$ & $44 \pm 2$ & $204 \pm 2$ & $\ldots$ & $\ldots$ & $1.7 \pm 0.2$ & $0.47 \pm 0.07$ \\
\hline
\end{tabular}

${ }^{a}$ Observation Date: JD minus 2,450,000

${ }^{\mathrm{b}}$ R. J. White et al. (2006, in preparation).

c System values for MHO-Tau-8 are determined from the mean and standard deviation for all HST observations; values for V410-Xray3 are determined from the long and short $z^{\prime}$ observations. 


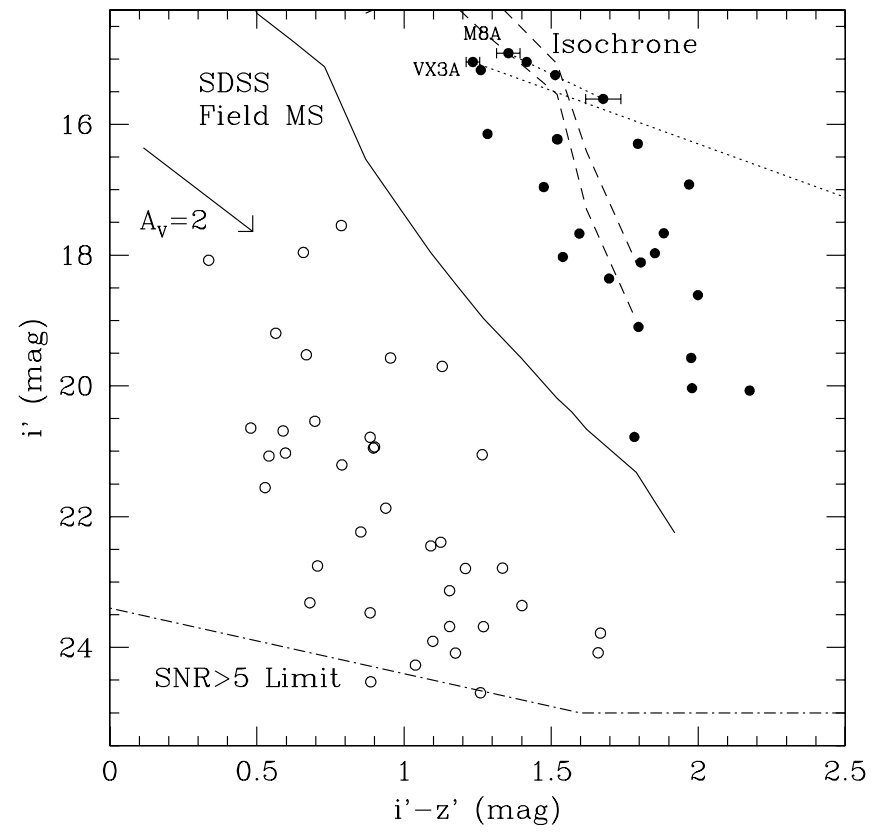

FIG. 4.-An $i^{\prime}$ vs. $i^{\prime}-z^{\prime}$ color-magnitude diagram. Taurus members are shown as filled circles; objects that are classified as background stars based on their position in this CMD are shown as open circles. The SDSS field main sequence (solid line), 1 and 2 Myr isochrones (dashed lines), and the detection limits of the survey (dot-dashed line) are also shown. The components of both binary systems are connected with a dotted line; the candidate secondary component of V410-Xray3 is well off the right side of the plot. The $i^{\prime}$ photometry for V410-Xray3 B is highly uncertain, so its $i^{\prime}-z^{\prime}$ color is not likely to be accurate. The statistical error bars for binary components are associated with each point; the error bars for single objects are smaller than the symbols, so they are not shown.

are most likely association members; based on their close proximity and the low density of association members, we conclude that these are physically associated companions. All other detected objects fall well below the empirical main sequence and are most likely background stars. One target object, KPNO-Tau-12, appears to be somewhat underluminous compared to the other Taurus VLMOs. The discovery survey (Luhman et al. 2003a) also found KPNO-Tau-12 to be underluminous for its assumed age and distance, so this is most likely a genuine feature of the system.

\subsection{Sensitivity Limits}

We determined detection limits as a function of distance from the primary stars via a Monte Carlo simulation similar to that of Metchev et al. (2003). We used the IRAF task DAOPHOT/ ADDSTAR and our average PSF to add artificial stars at a range of radial separations and magnitudes to the fields of GM Tau, KPNO-Tau-2, KPNO-Tau-7, and KPNO-Tau-4, which represent the full range of brightness in our sample. We then attempted to identify the artificial stars with the ALLSTAR PSF-fitting photometry package. Since the one confirmed VLMO binary in our sample (MHO-Tau-8) has already been resolved in other observations, our goal for these simulations was to determine the performance in retrieving known companions. Therefore, unlike similar simulations described in Kraus et al. (2005), we do not use DAOFIND to identify sources, but instead report the source positions directly to ALLSTAR for fitting. MHO-Tau-8 and V410Xray3 were the only targets that produced significant fits for two point sources, so these limits should be appropriate for the detection of new companions as well.

In Figure 5, we show the detection limits for the four representative targets in $V$ and $z^{\prime}$, as a function of separation, at which we can detect $>10 \%, 50 \%$, and $90 \%$ of the companions. At small separations ( $\$ 20 \mathrm{AU})$, the $50 \%$ detection thresholds roughly scale with the brightness of the primary; the survey limits are similar for all objects in terms of $\Delta m$. The detection limits converge to constant values at large separations; in the case where the noise is background dominated, our $5 \sigma$ detection limits are $z^{\prime}=23.8$, $i^{\prime}=25.1$, and $V=26.7$. The simulations demonstrate that we could identify bright, equal-mass pairs as close as 1 pixel $\left(0{ }^{\prime \prime} 027\right.$; $4 \mathrm{AU} ; \sim 0.5 \lambda / D)$ and binaries with mass ratio $q=0.1\left(\Delta z^{\prime}=4\right)$ at $\gtrsim 10$ AU. We also show the locations (in $\Delta m$ and separation) of the companion to MHO-Tau-8 and the candidate companion to V410-Xray3 (for $z^{\prime}$ only). These results indicate that the probability of detecting close binary companions in the $z^{\prime}$ images is near unity for even the faintest systems, but the probability of detecting similar systems in the $V$ filter declines rapidly for the faintest targets. Our inability to resolve the V410-Xray3 system in the $V$ band suggests a limit of $V>20.3$ for the secondary component; the corresponding color limit $\left(V-z^{\prime}>4.7\right)$ is consistent with the spectral type (M7.7) we determine in $\S 4.3$.

Our simulations suggest that it is not easier to resolve extremely red targets at shorter wavelengths; given the PSF stability of $H S T$, the improved number statistics at redder bands outweigh the superior diffraction-limited resolution at bluer bands. However, the additional color information was useful in confirming the identify of association members. Also, this is the first large set of homogeneous observations of young VLMOs shortward of $6000 \AA$. As we describe in $\S 4.3$, this allows us to characterize a previously unexplored regime in their spectral energy distributions.

\subsection{Uncertainties in Binary Properties}

A similar Monte Carlo routine was used to test the uncertainties in the measurements of MHO-Tau- 8 and V410-Xray3. We used ADDSTAR and the average PSF to construct 100 simulated images, given the positions and brightnesses reported for the primary and the secondary for the real images, and then used ALLSTAR to perform PSF-fitting photometry on these simulated images. The standard deviation in separation $(\sim 0.5$ mas $)$ reported for the simulated images of MHO-Tau- 8 is consistent with that calculated from the standard deviation in separation between the three filters ( $\sim 2$ mas), and the standard deviation in the flux ratio $\Delta m(0.04$ mag in each filter $)$ is consistent with that determined from the magnitudes reported by ALLSTAR for each object. The uncertainties predicted by our simulations are generally lower than those observed, but this is likely due to the small variations in PSF width observed for each target (§ 2.2). We found similar results for the $z^{\prime}$-band images of V410-Xray 3 ( 0.5 mas, $0.03 \mathrm{mag}$ ), but ALLSTAR only found a two-source fit for this object in $87 \%$ of the $i^{\prime}$-band images, and the standard deviation among successful fits was much higher in both separation ( $\sim 7$ mas) and flux ratio $(0.4 \mathrm{mag})$. These uncertainties are dominated by the measurements of the secondary component and match the uncertainties from the observations.

We also conducted Monte Carlo tests to determine the probability of mistakenly identifying a true single star as a binary. We constructed a series of simulated images (100 each for several objects spanning our sample's range of brightness) and then tried to fit each object with two point sources. We found that this never produced a successful fit, so the probability of an erroneous binary identification due to statistical errors appears to be low $(<1 \%)$. However, since the PSF width varies between observations, there is also a systematic component to the uncertainty in binary values. We have used the average PSF for all fits, but since there are no other bright objects in either binary field, it is 

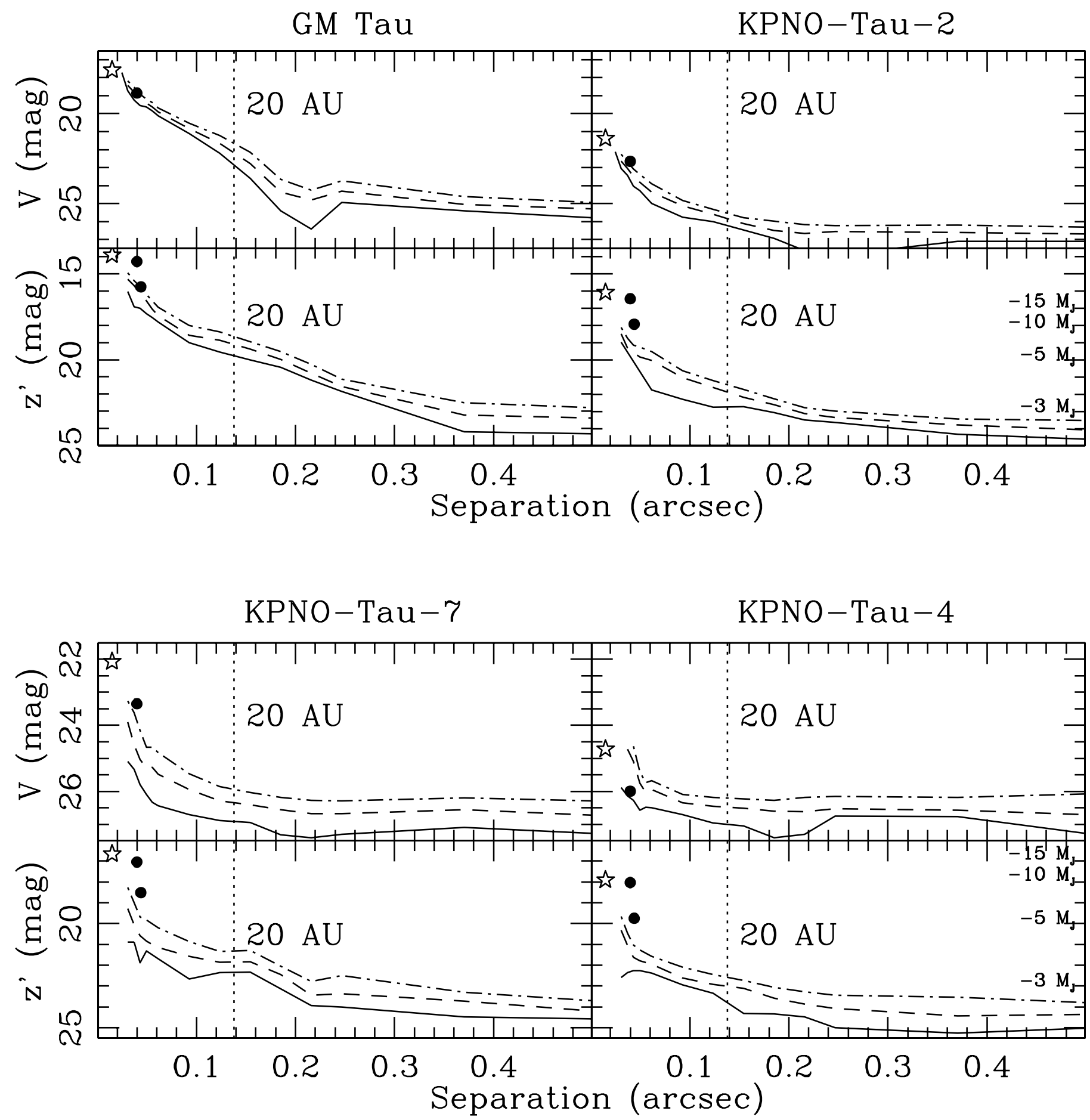

FIG. 5.-The $5 \sigma$ detection frequencies (10\%, solid line; $50 \%$, dashed line; $90 \%$, dash-dotted line) as a function of separation in each filter for single VLMOs of maximum, intermediate, and minimum brightness: GM Tau, KPNO-Tau-2 and KPNO-Tau-7, and KPNO-Tau-4, respectively. Corresponding brightnesses of potential planetary-mass companions (assuming $A_{V}=1.5$ ) are shown on the right for the $z^{\prime}$ plots. The brightness of the primary object is denoted with a star to allow conversion to $\Delta m$ values, and the vertical dotted line indicates a separation of $20 \mathrm{AU}$ at the distance of Taurus $(145 \mathrm{pc})$. The circles mark the separation and $\Delta m$ values for the binary companions to MHO-Tau- 8 and V410-Xray3.

not possible to determine whether our wide or narrow PSFs might be more appropriate in a specific case. Experiments show that the separation and position angle for each binary are independent of the choice of template PSF, but the photometry is not. If we attempt to fit the binaries with the wider template PSFs, then ALLSTAR is unable to produce a significant fit. If we attempt to fit the binaries with the narrower template PSFs, ALLSTAR reports values for $\Delta m$, which are systematically lower by $\sim 50 \%$.
This suggests that higher resolution observations will be required to determine accurate component fluxes.

\section{ANALYSIS}

\subsection{VLMO Binary Frequency}

For our 22 M5.75-M9.5 targets, we have confirmed a previously identified binary VLMO system and identified a new 
candidate binary, both with separations of $\sim 6$ AU. The observed binary fraction is thus either $4.5_{-1.4}^{+9.1} \%$ or $9.1_{-3.1}^{+9.9} \%$ for separations $\gtrsim 4$ AU. As can be seen in Figure 5, the detection of faint companions is difficult at separations comparable to the PSF width (58 mas in $V$ ). Consequently, the total binary fraction in this separation range may be higher. However, studies of VLMO binaries in the field and in Upper Scorpius have found that companions preferentially have mass $\operatorname{ratios} q>0.6$ despite the surveys' sensitivity to companions with lower mass ratios (Close et al. 2003; Bouy et al. 2003; Kraus et al. 2005), which corresponds to $\Delta z<1$ according to the models of Baraffe et al. (1998). If Taurus VLMOs have a similar mass ratio distribution, then the detection thresholds in Figure 5 predict that we should identify all VLMO companions at separations $\gtrsim 4 \mathrm{AU}$ in at least $z^{\prime}$ and likely in the other filters as well. Even if these limits are optimistic, the identification of V410-Xray3 as a candidate binary near the minimum measurable separation, $\sim 5 \mathrm{AU}$, implies that the survey should be complete to $\Delta z^{\prime}=2(q=0.45)$ for separations $\gtrsim 4 \mathrm{AU}$. It is therefore unlikely that there are many additional binaries at separations $\gtrsim 4$ AU with $q>0.45$ in this sample.

The intrinsically higher luminosity of binaries makes them easier to identify in membership surveys, which can also bias the binary frequency to larger values. However, MHO-Tau- 8 and V410-Xray3 are substantially brighter than the detection limits of their discovery surveys and would have been easily detected without the additional flux of their companions, so this effect should not bias our multiplicity results.

\subsection{Limits on Planetary-Mass Companions}

The high dynamic range of our deep exposures would allow us to directly image wide planetary-mass companions. In Figure 5, we indicate the predicted brightness of some representative masses of planetary companions based on the 1 Myr DUSTY models of Chabrier et al. (2000). These values assume an extinction of $A_{V} \sim 1.5$, a typical value for objects in Taurus. The extremely red colors predicted for planetary-mass objects imply that $z^{\prime}$ observations provide the strictest limits on planetary companions. Based on the lack of detections, we conclude that there are no planetary companions with mass $\gtrsim 3 M_{\mathrm{J}}$ at projected separations larger than 280 mas (40 AU) for the brightest targets or 140 mas (20 AU) for the faintest targets. For comparison, these limits would have allowed for a significant detection of the planetary-mass companion to 2MASSW J1207334-393254, a substellar member of the TW Hya association, which has a projected separation of $\sim 54 \mathrm{AU}$, a flux ratio of $\Delta z^{\prime} \sim 7$, and a predicted mass of $\sim 5 M_{\mathrm{J}}$ (Chauvin et al. 2004). Other surveys in Cha I (12 targets; Neuhauser et al. 2002) and IC 348 (37 targets; Luhman et al. $2005 \mathrm{a})$ that included VLMOs in the same mass range $\left(\lesssim 0.12 M_{\odot}\right)$ also found no planetary-mass companions.

\subsection{Inferred (Sub)stellar Properties}

\subsubsection{Masses and Spectral Types}

In Table 4, we give the inferred spectral types and masses for all of the VLMOs in our sample. Spectral types for single VLMOs and for binary primaries in our sample are taken from the discovery sources listed in Table 1 and were determined via low- or intermediate-resolution spectroscopy. The masses for this sample are estimated from the 2 Myr mass-magnitude-temperature relations of Baraffe et al. (1998) and the temperature-spectral type relations of Luhman et al. (2003b) and range from 0.015 to $0.14 M_{\odot}$. Large systematic errors may be present in these and all pre-mainsequence models (e.g., Baraffe et al. 2002; Hillenbrand \& White
2004; Close et al. 2005; Reiners et al. 2005), so they are best used for relative comparison only.

The models of Baraffe et al. predict that the mass ratio $(q=$ $\left.m_{s} / m_{p}\right)$ and difference in component spectral types $(\Delta \mathrm{SpT})$ for young VLMO binaries are a function of the primary-to-secondary flux ratios $\Delta m$ with only a minor mass dependence; we report these quantities as determined from the flux ratio $\Delta z^{\prime}$ in Table 3 for the detected binary VLMOs, MHO-Tau-8 and V410-Xray3. The models do not report $z^{\prime}$ magnitudes, so we calculated these from the model $I_{\mathrm{C}}$ magnitudes, the $I_{\mathrm{C}}-i^{\prime}$ transformations determined in $\S 2$, and the $i^{\prime}-z^{\prime}$ colors for the appropriate spectral type. The uncertainties reflect the uncertainties in the flux ratios and the scatter in this relation in the models, but do not include any systematic uncertainties from the models. The values determined from $\Delta V$ and $\Delta i^{\prime}$ for MHO-Tau- 8 are roughly consistent with these results, but the values for $\Delta z^{\prime}$ are the only reliable result for V410-Xray3, so we use $\Delta z^{\prime}$ in all cases for uniformity.

\subsubsection{Luminosities and Extinctions}

Our results, in combination with other surveys, also allow us to determine the luminosity and extinction for each VLMO by fitting the observed spectral energy distribution (SED) with a reddened dwarf SED of the same spectral type. This allows us to test for potential optical excesses that previous observations at longer wavelengths would not have detected. The VLMO SEDs are constructed from $V i^{\prime} z^{\prime} J H K$ photometry from this work and from the Two Micron All Sky Survey (2MASS; Cutri et al. 2003). Our standard SEDs are constructed from the mean $i^{\prime} z^{\prime} J H K$ photometry for field stars in the SDSS (West et al. 2005) and 2MASS (Leggett et al. 2002) plus the mean $V-K$ colors reported for the field by Reid et al. (2004). Typical uncertainties are $\lesssim 0.05$ mag for our observations and $\sim 0.1-0.2$ mag for the field star photometry. Any significant difference between a VLMO SED and the corresponding field SED should be a result of the intrinsically higher luminosity of our targets (pre-main-sequence VLMOs have larger radii) or extinction due to obscuring material. These will result in a constant multiplication and a wavelength-dependent multiplication to the flux in each band, respectively. We solve for these constants, which correspond to $L / L_{\mathrm{MS}}$ and $A_{V}$, by a least-squares fit of the set of equations

$$
m_{i, \mathrm{VLMO}}=m_{i, \text { ield }}+\frac{A_{i}}{A_{V}} A_{V}-2.5 \log \frac{L}{L_{\mathrm{MS}}},
$$

where $i$ denotes the five filters used in the fit $\left(i^{\prime} z^{\prime} J H K\right)$ and the reddening coefficient for each band is taken from Schlegel et al. (1998). Variations in distance will also be included in the luminosity term, but if the depth is similar to the apparent width of Taurus-Auriga on the sky $\left(\sim 10^{\circ} ; 25 \mathrm{pc}\right)$, these should be relatively minor.

We list the inferred luminosity ratio, total luminosity, radius, and extinction for each Taurus VLMO in Table 4. The total luminosity is determined from the $K$-band magnitude, corrected for extinction, and the bolometric corrections of Leggett et al. (2002), which are $\mathrm{BC}_{K} \sim 3.1$ for mid- to late $\mathrm{M}$ dwarfs. The radius is determined from the luminosity ratio $\left[L / L_{\mathrm{MS}} \propto\left(R / R_{\mathrm{MS}}\right)^{2}\right]$ and the main-sequence mass-radius relations of Baraffe et al. (1998), which find that $R_{\mathrm{MS}} / R_{\odot} \sim M_{\mathrm{MS}} / M_{\odot}$ for M5-M9 dwarfs at ages $>1$ Gyr. We also performed some experiments in varying the spectral type of the field standard; these imply that the uncertainty in spectral type (typically half a subclass) corresponds to uncertainties of $\sim 0.5$ in $A_{V}, \sim 20 \%$ in $L / L_{\mathrm{MS}}$, and $\sim 10 \%$ in $R$. The uncertainty in the bolometric correction is typically $\sim 5 \%$ for spectral 
TABLE 4

VlMO (Sub)stellar Properties

\begin{tabular}{|c|c|c|c|c|c|c|c|c|}
\hline Name & $\mathrm{SpT}^{\mathrm{a}}$ & $\begin{array}{c}M \\
\left(M_{\odot}\right)^{\mathrm{b}}\end{array}$ & $A_{V}$ & $L / L_{\mathrm{MS}}$ & $\begin{array}{l}\log L \\
\left(L_{\odot}\right)\end{array}$ & $\begin{array}{c}R \\
\left(R_{\odot}\right)\end{array}$ & Accretor? $^{\mathrm{c}}$ & $E\left(V-V_{\mathrm{MS}}\right)$ \\
\hline CFHT-Tau-1 .......................... & M7 & 0.055 & 4.8 & 54 & -1.4 & 0.73 & $\mathrm{~N}$ & -0.59 \\
\hline CFHT-Tau-2 …..................... & M7.5 & 0.04 & 2.7 & 35 & -1.6 & 0.56 & $\mathrm{~N}$ & -0.35 \\
\hline CFHT-Tau-3 ......................... & M7.75 & 0.035 & 1.8 & 29 & -1.8 & 0.50 & $\mathrm{~N}$ & -0.71 \\
\hline 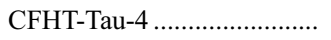 & M7 & 0.055 & 4.4 & 210 & -0.8 & 1.45 & $Y ?$ & -0.61 \\
\hline KPNO-Tau-1 ........................ & M8.5 & 0.02 & 1.5 & 10 & -2.3 & 0.27 & $\mathrm{~N}$ & 0.08 \\
\hline KPNO-Tau-2 ...................... & M7.5 & 0.04 & 0.7 & 17 & -2.0 & 0.39 & $\mathrm{~N}$ & -0.25 \\
\hline KPNO-Tau-3 ...................... & M6 & 0.1 & 2.0 & 18 & -1.6 & 0.47 & $\mathrm{Y}$ & -0.74 \\
\hline KPNO-Tau-4 ...................... & M9.5 & 0.01 & 4.0 & 31 & -2.0 & 0.42 & $Y ?$ & -1.18 \\
\hline KPNO-Tau-5 ........................ & M7.5 & 0.04 & 0.3 & 51 & -1.5 & 0.68 & $\mathrm{~N}$ & -0.35 \\
\hline 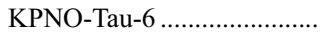 & M8.5 & 0.02 & 0.7 & 9 & -2.3 & 0.26 & $\mathrm{Y}$ & -0.95 \\
\hline KPNO-Tau-7 ........................ & M8.25 & 0.017 & 0.1 & 11 & -2.2 & 0.29 & $\mathrm{Y}$ & -0.12 \\
\hline KPNO-Tau-8 ..................... & M5.75 & 0.11 & 0.7 & 15 & -1.7 & 0.44 & $\mathrm{~N}$ & -0.02 \\
\hline KPNO-Tau-9 ........................... & M8.5 & 0.02 & 1.7 & 7 & -2.5 & 0.22 & $\mathrm{~N}$ & 0.43 \\
\hline KPNO-Tau-12 ...................... & M9 & 0.014 & 1.6 & 3 & -2.8 & 0.14 & $\mathrm{Y}$ & -2.15 \\
\hline KPNO-Tau-14 ................... & M6 & 0.1 & 4.2 & 123 & -0.8 & 1.22 & $\mathrm{~N}$ & -0.42 \\
\hline MHO-Tau-4 ......................... & M7 & 0.055 & 0.8 & 126 & -1.1 & 1.12 & $\mathrm{~N}$ & -0.39 \\
\hline MHO-Tau-5 .......................... & M7 & 0.055 & 0.3 & 191 & -0.9 & 1.38 & $Y ?$ & -0.39 \\
\hline MHO-Tau-8 AB .................. & M6+M6.6 & 0.17 & 1.7 & 158 & -0.7 & 1.38 & $\mathrm{~N}$ & -0.38 \\
\hline 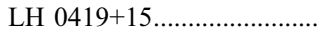 & M7 & 0.055 & 1.2 & 12 & -2.1 & 0.35 & $\ldots$ & -0.22 \\
\hline V410 Xray-3 AB ................. & M6+M7.7 & 0.14 & 1.5 & 81 & -1.0 & 0.99 & $\mathrm{~N}$ & -0.54 \\
\hline 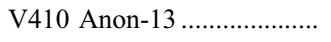 & M5.75 & 0.11 & 5.6 & 60 & -1.0 & 0.87 & $\mathrm{Y}$ & -0.57 \\
\hline 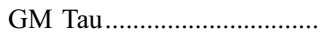 & M6.5 & 0.08 & 0.2 & 49 & -1.1 & 0.74 & $\mathrm{Y}$ & -1.23 \\
\hline 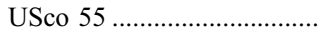 & M5.5 & 0.010 & 0.1 & 18 & -1.5 & 0.49 & $\mathrm{~N}$ & 0.18 \\
\hline USco $66 \ldots \ldots \ldots$. & M6 & 0.07 & -0.4 & 16 & -1.7 & 0.44 & $\mathrm{~N}$ & -0.58 \\
\hline 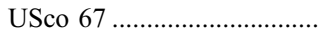 & M5.5 & 0.10 & 0.4 & 18 & -1.5 & 0.49 & $\mathrm{~N}$ & 0.25 \\
\hline 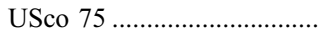 & M6 & 0.07 & 0.2 & 19 & -1.6 & 0.48 & $\mathrm{~N}$ & -0.43 \\
\hline 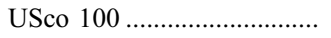 & M7 & 0.05 & -0.2 & 34 & -1.6 & 0.58 & $\mathrm{~N}$ & -0.40 \\
\hline 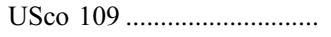 & M6 & 0.057 & 0.4 & 9.1 & -1.9 & 0.33 & $\mathrm{~N}$ & -0.33 \\
\hline 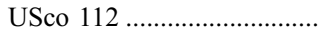 & M5.5 & 0.10 & -0.2 & 6.8 & -1.9 & 0.30 & $\mathrm{~N}$ & -0.63 \\
\hline USco $128 \ldots \ldots \ldots \ldots \ldots \ldots \ldots \ldots . .$. & M7 & 0.05 & 0.5 & 10 & -2.2 & 0.32 & $\mathrm{~N}$ & -0.28 \\
\hline 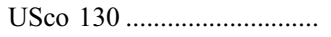 & M7.5 & 0.04 & 0.4 & 12 & -2.1 & 0.33 & $\mathrm{~N}$ & -0.28 \\
\hline USco 131 ............................ & M6.5 & 0.06 & 1.3 & 6.7 & -2.2 & 0.27 & $\mathrm{~N}$ & -0.08 \\
\hline 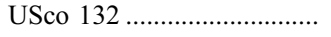 & M7 & 0.05 & 1.9 & 14 & -2.0 & 0.37 & $\cdots$ & -0.40 \\
\hline USco 137 ............................. & M7 & 0.05 & 1.0 & 3.3 & -2.6 & 0.18 & $\ldots$ & -0.28 \\
\hline
\end{tabular}

${ }^{\text {a }}$ Spectral types for binary secondaries are inferred from photometry presented here; others are from their discovery source ( $(4.3)$.

${ }^{\mathrm{b}}$ Masses are determined from the models of Baraffe et al. (1998).

${ }^{\mathrm{c}}$ Muzerolle et al. (2003, 2005); Mohanty et al. (2005).

types later than M5 (Leggett et al. 2002). The statistical uncertainties from the fitting process are generally insignificant compared to these systematic uncertainties. These experiments also reveal a degeneracy in the determination of extinction and luminosity; fitting with an earlier or later spectral type yields systematically different extinctions and luminosities, but the goodness offit does not decrease significantly for small changes ( $\lesssim 1$ subclass). The same fitting process has been applied to the binaries by considering the total system brightness in each filter and assigning it the spectral type of the primary, fitting it with a field standard corresponding to the spectral type of the primary. However, since the observed spectrum is a composite of both objects' spectra, the results may be biased.

In Figure 6, we present SEDs for the 18 Taurus VLMOs that produced successful fits to the dwarf SEDs (within $2 \sigma$ ). Four stars could not be fit well and are discussed below. The solid lines and filled circles denote VLMO SEDs that have been corrected for extinction, and the dashed lines denote the best-fit dwarf SEDs that have been shifted upward by $\log \left(L / L_{\mathrm{MS}}\right)$ to correct for their intrinsically lower luminosity. Typical uncertainties in the SED flux measurements are $\sim 0.02$ dex for the VLMO SEDs, $\sim 0.05 \mathrm{dex}$ for the $r^{\prime} i^{\prime} z^{\prime} J H K$ fluxes in the dwarf SEDs, and $\sim 0.15$ dex for the $V$-band fluxes in the dwarf SEDs. Since our results typically agree with the dwarf SEDs to within the uncertainties, the common assumption of dwarf colors for premain-sequence objects appears to be valid for this age and mass regime. However, as we discuss in $\S 4.3 .3$, the targets appear to be systematically brighter than equivalent dwarf SEDs in $V$, yielding bluer optical colors. In Figure 7, we present SEDs for the four VLMOs with significant discrepancies in their fit: KPNO-4, KPNO-6, KPNO-12, and GM Tau. The first three objects deviate significantly only in their $V$-band measurement, but the optical and near-infrared portions of the SED for GM Tau appear to be inconsistent; we cannot produce a fit to its dwarf reference by any single combination of $A_{V}$ and $L / L_{\mathrm{MS}}$. Based on the slope of its NIR SED, GM Tau appears to have been significantly fainter and/or possessed a significant $K$-band excess during its epoch of observation in 2MASS. The excellent agreement between the optical and near-infrared values for all other objects, which were taken at different epochs, suggest that they are typically not variable at amplitudes of $>0.2 \mathrm{mag}$.

\subsubsection{Optical Excesses}

Nine of the VLMOs in our sample have been observed to possess spectroscopic signatures of active accretion of circumstellar material by Muzerolle et al. $(2003,2005)$ and Mohanty et al. 


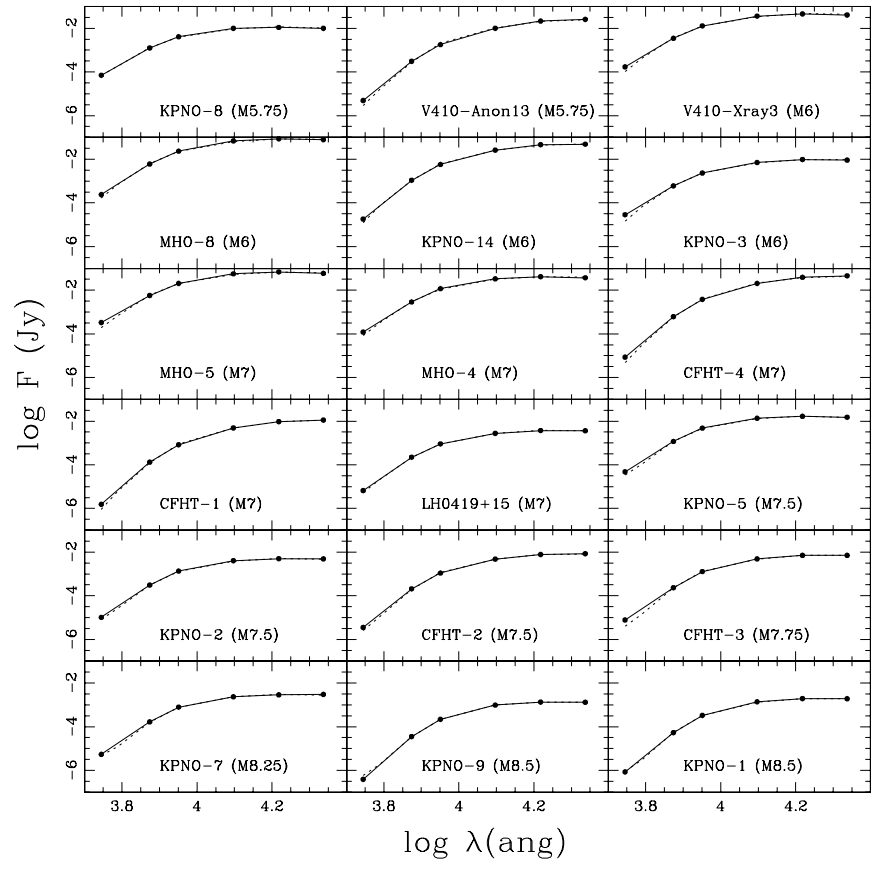

FIG. 6.- Spectral energy distributions for the VLMOs in our Taurus sample that produced successful fits. Solid lines and circles denote the target SEDs, which have been corrected for extinction. Dashed lines denote field SEDs, which have been corrected for the higher luminosity of our targets.

(2005). Accreted material falling onto more massive T Tauri stars is typically heated to higher temperatures than the stellar photosphere, leading to an optical excess (Basri \& Batalha 1990; Hartigan et al. 1991). However, since the rate of accretion appears to depend on system mass (e.g., Muzerolle et al. 2003), the low mass accretion rates of T Tauri VLMOs should not produce any significant excess. Nonetheless, as we note in $\S 4.3 .2$, several VLMOs show signs of an optical excess, which might be a result of accretion. In Table 4, we identify the known accretors and nonaccretors and list the $V$-band excess or deficit (in magnitudes) observed for each target. The four objects with the most significant $V$-band discrepancies are confirmed or probable accretors.

In Figure 8, we show a plot of the $V$-band excesses and deficits as a function of spectral type for each target. Filled circles denote Taurus VLMOs; symbols for known accretors (based on $\mathrm{H} \alpha$ or

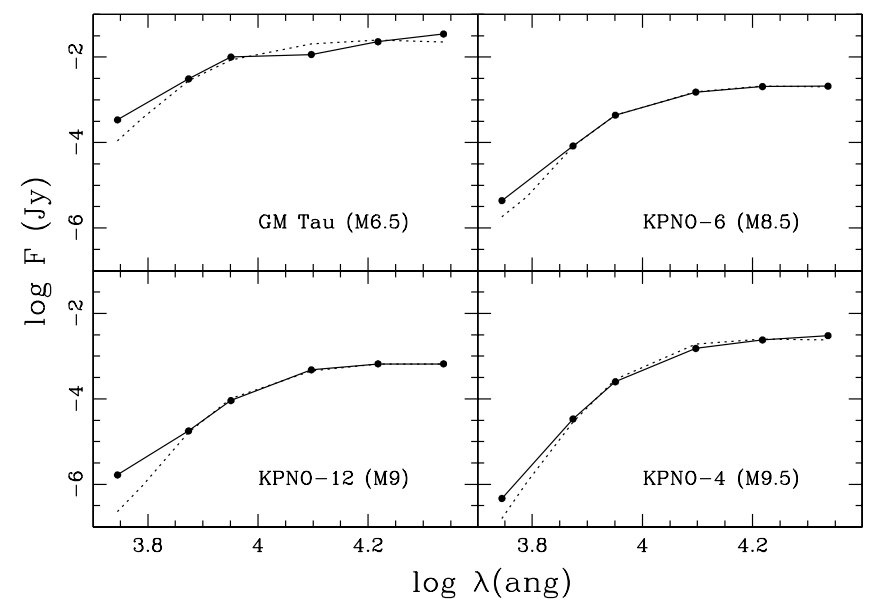

FIG. 7.- Spectral energy distributions for the four VLMOs in our sample that produced anomalous fits.

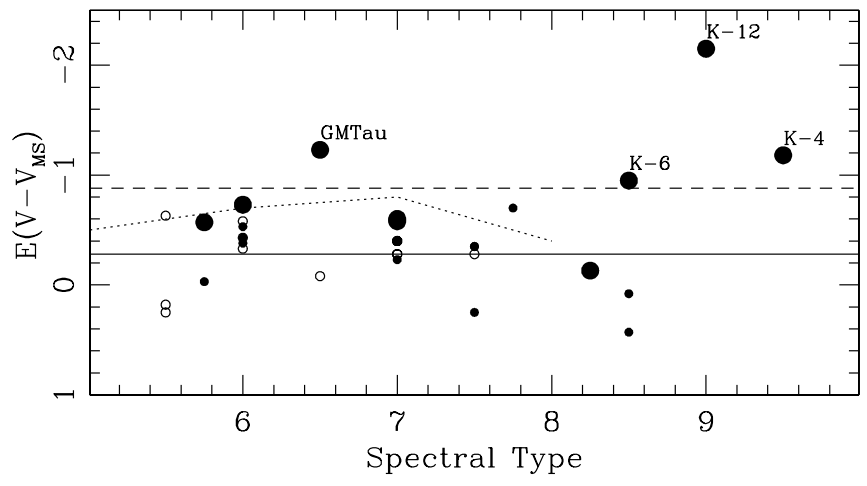

FIG. 8.- $V$-band excesses as a function of spectral type. Filled circles denote Taurus members, open circles denote Upper Sco members, and large symbols denote confirmed or probable accretors. The solid line shows the mean value for all nonaccretors in our sample, and the dashed line shows the corresponding $2 \sigma$ limit for the existence of significant optical excesses. The four objects with significant excesses are labeled.

other line profile diagnostics) are larger than those of nonaccretors. Open circles denote Upper Sco VLMOs, none of which are known accretors. The dotted line denotes the $V$-band excess one would mistakenly infer if the color were influenced by surface gravity effects, estimated here using the intrinsically bluer $V-I$ colors of M5-M8 giants (Bessell \& Brett 1988). Most of the known accretors are located preferentially higher in the plot relative to the nonaccretors and relative to the giant locus, implying that they possess intrinsic excesses, which are related to accretion or to the assessment of their accretion status.

Even nonaccretors seem to possess small $V$-band excesses over typical dwarf colors, although they are not as high as typical giant excesses and do not depend significantly on spectral type; this suggests that young VLMOs tend to be bluer than dwarfs of the same spectral type. The mean and standard deviation for this discrepancy among known nonaccretors are $0.28 \pm 0.33$ mag; in Figure 8 , the mean value is denoted with a solid line, and the $+2 \sigma$ limit with a dashed line. If we regard this mean value as a measure of the true photospheric flux for young VLMOs, then the four objects identified earlier still possess excesses significant at $\gtrsim+2 \sigma$.

In Table 5, we present the (sub)stellar properties for our Upper Sco sample (Kraus et al. 2005), which we derive using the methods described in $\S 4.3$. There are no known accretors, and the mean $V$-band excess $(0.27 \pm 0.08)$ is consistent with the mean excess for nonaccretors in Taurus $(0.28 \pm 0.10)$. As we show in Figure 8, all Upper Sco VLMOs fall below the $+2 \sigma$ criterion for a statistically significant optical excess over the nonaccretor value. This supports the suggestion that accretors may show optical excesses over nonaccretors, although the older age of Upper Sco may also play a role.

\section{DISCUSSION}

\section{1. (Sub)stellar Multiplicity}

Multiplicity surveys of VLMOs have suggested a fairly uniform set of binary properties in the field (Close et al. 2003; Bouy et al. 2003; Burgasser et al. 2003; Siegler et al. 2005), in the Pleiades (Martin et al. 2003), and in the young OB association Upper Scorpius (Kraus et al. 2005). No companions with wide separations $(>20 \mathrm{AU})$ or with unequal mass ratios $(q<0.6)$ were found, despite sufficient sensitivity for their detection. Several wider systems have been discovered serendipitously or during ongoing surveys (Gizis et al. 2000; Luhman 2004a, 2005; Phan-Bao 
TABLE 5

Results for Previous Multiplicity Surveys

\begin{tabular}{|c|c|c|c|}
\hline Survey & SpT & $\begin{array}{c}\text { Minimum Separation } \\
(\mathrm{AU})\end{array}$ & $\begin{array}{c}\text { Binary Frequency } \\
(\%)\end{array}$ \\
\hline 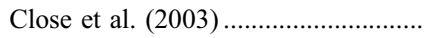 & M8.0-L0.5 & 3 & $15 \pm 7$ \\
\hline 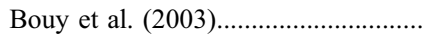 & M8.0-L8.0 & 1 & $18.8 \pm 3.7$ \\
\hline Burgasser et al. (2003) ........................ & T5.0-T8.0 & 1 & $9_{-3}^{+4}$ \\
\hline 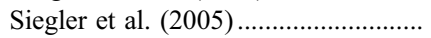 & M6.0-M7.5 & 3 & $9_{-3}^{+4}$ \\
\hline 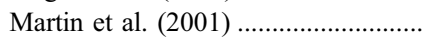 & M6.0-M9.5 ${ }^{\mathrm{a}}$ & 7 & $15_{-5}^{+15}$ \\
\hline 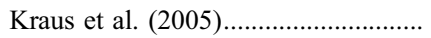 & $\mathrm{M} 5.5-\mathrm{M} 7.5^{\mathrm{a}}$ & 5 & $25_{-9}^{+16}$ \\
\hline Current survey & $\mathrm{M} 5.75-\mathrm{M} 9.5^{\mathrm{a}}$ & 5 & $9_{-4}^{+10}$ \\
\hline
\end{tabular}

${ }^{\text {a }}$ The surveys by Martin et al. and Kraus et al. observed BDs in the Pleiades and Upper Sco, respectively. Since these regions are young, the spectral type range corresponds to lower masses than in the field.

et al. 2005; Billeres et al. 2005). However, the corresponding survey statistics are not available, so it is difficult to determine a frequency for wide VLMO binaries. The binary fractions observed were also significantly lower than the binary fractions of $57 \%$ for field G dwarfs (Duquennoy \& Mayor 1991) and 35\%-43\% for field M dwarfs (Reid \& Gizis 1997; Fischer \& Marcy 1992). We summarize these results in Table 5.

The study presented here is the first large survey of young VLMOs in Taurus-Auriga with sufficient resolution to identify binaries at separations seen for field VLMOs. Our confirmed binary fraction, $4.5_{-1.4}^{+9.1} \%$ for separations $\gtrsim 4 \mathrm{AU}$, is lower than, but marginally consistent, with the results cited for the field. If the binary candidate V410-Xray3 is confirmed, the binary fraction in this separation range would then be $9.1_{-3.1}^{+9.9} \%$, which falls within the range of results seen in the field. However, the field surveys are sensitive to companions with separations as small as $\sim 1 \mathrm{AU}$ and found many companions within $4 \mathrm{AU}$ that we could not have detected because of the larger distance to our targets. Our binary fraction for companions with $a \gtrsim 4$ AU only sets a lower limit on the total binary fraction for VLMOs. The mass ratio and separation for MHO-Tau-8 $(q=0.7, a \sim 6 \mathrm{AU})$ and the separation for V410-Xray3 ( $\sim 6 \mathrm{AU})$ are consistent with the field, but the mass ratio for V410-Xray3 may be substantially less. However, this result is based only on the $z^{\prime}$ observations and should be confirmed with follow-up observations.

The methodology of this survey is identical to our Upper Scorpius survey, and their similar distances imply similar spatial resolution, so the two results can be combined to produce a total VLMO binary fraction (either 4 or 5 of 34 , corresponding to $12_{-4}^{+8} \%$ or $15_{-4}^{+8} \%$ ), which is also consistent with the field. Our binary fraction for Taurus $\left(9_{-3}^{+10} \%\right)$ is somewhat lower than that for Upper Scorpius $\left(25_{-9}^{+16} \%\right)$, although the difference is not statistically significant $(<2 \sigma)$. However, larger surveys (particularly in Upper Sco) will be required to test whether the binary fraction depends on mass.

\subsection{The Mass Dependence of Multiplicity}

The distinct binary properties of field BDs, relative to those of stars, indicate that these properties are mass dependent. However, the form of this dependence is not known; Kroupa et al. (2003), Close et al. (2003), and Kraus et al. (2005) interpret current data as a sharp transition near the stellar/substellar boundary, while Luhman (2004b) and R. J. White et al. (2006, in preparation) argue for a smooth mass dependence. The field binary fraction appears to decrease from $57 \%$ for $\mathrm{G}$ dwarfs to $35 \%-43 \%$ for M dwarfs (Duquennoy \& Mayor 1991; Fischer \& Marcy 1992; Reid \& Gizis 1997), but the trend with mass is only marginally significant.
Our combined young VLMO sample for Taurus and Upper Scorpius is large enough to investigate a possible mass dependence in the VLMO binary frequency over the single order of magnitude mass range of our survey $\left(0.015-0.12 M_{\odot}\right)$. All five binaries and candidate binaries were found among the 13 most massive VLMO targets $\left(M \gtrsim 0.07 M_{\odot}\right)$; none were found among the 21 lower mass VLMOs. If the probability of binarity is $5 / 34$ for all members of our sample, then the probability that all five binaries will fall among the 13 most massive targets is (13!/8!)/ $(34 ! / 29$ ! $) \sim 0.005$. The possible double-lined spectroscopic binary KPNO-Tau-14 (Mohanty et al. 2005) also falls in the upper mass range, although its separation is well inside our survey limit. The detection limits in Figure 5 demonstrate that bright binaries are more easily identified due to superior photon statistics, but at least the three high-confidence binaries in the combined sample (USco-55, USco-66, and MHO-Tau-8) could have been identified around even the faintest targets. Also, since this trend is seen at lower significance in both regions, it should be robust against variations in initial conditions. The implication is that the binary fraction may decline over the mass range, although a constant binary fraction combined with a shift toward smaller separations could produce the same effect in our resolution-limited sample.

We can further investigate the mass dependence of multiplicity in Taurus by combining our results with a binary census of more massive Taurus members by R. J. White et al. (2006, in preparation). This speckle interferometry survey included 44 members of Taurus in the mass range $0.06 M_{\odot}<M<1.5 M_{\odot}$ and, in combination with previous results for 41 additional targets (Leinert et al. 1993; Ghez et al. 1993; Simon et al. 1995; Duchenne 1999), identified 23 binaries with separations $>27$ mas (4 AU). The sample is reported to be complete for separations of 9-460 AU and mass ratios $>0.1(\Delta K<2.75)$, which fall within the completeness range of our survey $(\S 3.3)$. They report that the binary frequency appears to peak for stars with masses of $0.3-$ $0.7 M_{\odot}$ and decline toward lower masses, although the results are not statistically significant $(<2 \sigma)$. Our VLMO sample supports this mass dependence, since we found no binaries with separations in the completeness regime. However, as discussed in Kraus et al. (2005), a similar survey in Upper Scorpius by Kohler et al. (2000) found no evidence of a similar decline until immediately above the stellar/substellar boundary $\left(0.1-0.2 M_{\odot}\right)$. This implies that the mass dependence of multiplicity could also have a regional dependence.

\subsection{A Future Dynamical Mass for MHO-Tau-8?}

Theoretical models of the mass-luminosity-temperature relation are still largely uncalibrated for low masses and young 
ages (e.g., Baraffe et al. 2002). Surface gravity measurements for young brown dwarfs in Upper Sco (Mohanty et al. 2005) have provided some evidence that the theoretical models underestimate masses for very low mass stars and brown dwarfs and overestimate masses for the least massive brown dwarfs, but the measurement of dynamical masses for low-mass binaries will be required to directly calibrate the models. To date, this has been done only for three young VLMOs, the older ( 50-100 Myr) brown dwarf AB Dor C (Close et al. 2005; Luhman et al. 2005b), and both components of the young substellar eclipsing binary 2MASS J05352184-0546085 (Stassun et al. 2006).

Relative to the speckle interferometry measurement by R. J. White et al. (2006, in preparation), MHO-Tau- 8 B has traced $\sim 40^{\circ}$ of its orbit around MHO-Tau- 8 A within only $\sim 5 \mathrm{yr}$; this suggests an orbital period of $\sim 45 \mathrm{yr}$. If we assume a circular faceon orbit, then we estimate from Kepler's law and the system parameters ( $a \gtrsim 6 \mathrm{AU}, P \sim 45 \mathrm{yr}$ ) that the total mass of the system is $\gtrsim 0.11 M_{\odot}$, which is consistent with the total system mass implied by the models of Baraffe et al. (1998), $\sim 0.17 M_{\odot}$. Since this lower limit is substantially lower than the predicted total mass, the orbit of the secondary is likely either inclined relative to the plane of the sky (implying a larger semimajor axis) or highly eccentric and near apastron. In either case, additional astrometric measurements for this system should yield precise orbital parameters and better limits on the dynamical masses of its components over the next decade.

\subsection{VLMO Optical Excesses}

The rate of (sub)stellar mass accretion onto $\mathrm{T}$ Tauri stars is typically estimated either by measuring the optical continuum excess or by modeling its effect on emission line features that result from outflows as accreting material impacts the photosphere. Previous observations of brown dwarfs and low- and intermediatemass stars have found mass accretion rates that scale roughly as $\dot{M} \propto M^{2}$ for masses of 0.03-3.0 $M_{\odot}$ (Gullbring et al. 1998; White \& Ghez 2001; Muzerolle et al. 2003; Calvet et al. 2004). Specifically, these surveys found mass accretion rates ranging from $\sim 10^{-7} M_{\odot} \mathrm{yr}^{-1}$ for intermediate-mass T Tauri stars with mass 3 to $\sim 10^{-11} M_{\odot} \mathrm{yr}^{-1}$ for T Tauri-age brown dwarfs. Recent results from Mohanty et al. (2005) and Muzerolle et al. (2005), who measure accretion rates by modeling of $\mathrm{Ca}$ II and $\mathrm{H} \alpha$ emission, respectively, support this trend. They found that many low-mass brown dwarfs (including KPNO-6, KPNO-12, and possibly KPNO-4) have apparent mass accretion rates of $\sim 5 \times 10^{-12} M_{\odot} \mathrm{yr}^{-1}$.

These low accretion rates would result in negligible optical excesses $\left[E\left(V-V_{\mathrm{MS}}\right)<0.01\right.$; e.g., Muzerolle et al. 2003]; the very large excesses observed for our targets imply that either these objects have much higher accretion rates or the $V$-band excesses are not the result of accretion. The mass accretion rates that would be required for each known accretor to generate the optical excess can be calculated using the method described in Gullbring et al. (1998) whereby the excess $V$-band flux is converted to a total accretion luminosity. This luminosity is then assumed to result from mass infall from the inner edge of a circumstellar disk onto the surface of the star via magnetospheric accretion. Specifically, we assume that the radius of the inner disk edge is $5 R_{\mathrm{VLMO}}$ and the entire change in potential energy is radiated away via the optical excess. To correct our $V$-band excess luminosity to the total accretion luminosity, we adopt a bolometric correction factor of 10 , which is $10 \%$ less than the bolometric correction adopted by White \& Hillenbrand (2004) for a slightly redder, but narrower bandpass $(0.60-0.65 \mu \mathrm{m})$. As emphasized by White \& Hillenbrand, these bolometric corrections are still highly uncertain, but by adopting a value consistent with previous assumptions, we can minimize sys- tematic errors in comparisons with previous calculations. Unfortunately, there are no photometric or spectroscopic data available at wavelengths shorter than $6000 \AA$ for young BDs, so our results cannot be directly compared to any in the literature.

The resulting mass accretion rates for the four stars with significant excesses $\left(10^{-7}\right.$ to $\left.10^{-9} M_{\odot} \mathrm{yr}^{-1}\right)$ are 3-4 orders of magnitude higher than the values found via other methods or inferred from the $M-\dot{M}$ relations observed for higher mass T Tauri stars. Given this discrepancy, we consider the possibility that the excess results from our analysis methods or from other physical processes. However, it is statistically unlikely that $4 / 22$ objects would possess $>+2 \sigma$ excesses or that KPNO-12 would possess a $6 \sigma$ excess, if these accretors are part of a random distribution with the same mean and standard deviation as we observe for nonaccretors. Since $4 / 9$ accretors possess excesses significant at $>+2 \sigma$ and $8 / 9$ possess excesses significant at $\gtrsim+1 \sigma$, it appears that the $V$-band excesses are related to accretion diagnostics. Since three of the outliers fall among the five latest objects, there could also be a gravity-based $V$-band excess inherent in all late-type young VLMOs. However, the corresponding trend for giants (which possess even lower surface gravity) appears to decline for spectral types later than M7 (Fig. 8), and there is no sign of a similar excess for the two nonaccreting late-type objects, KPNO-1 and KPNO-9. Finally, the possibility of erroneous spectral classifications cannot explain this result because there are significant discrepancies in the SED fits for errors of more than one subclass, and the degeneracy between spectral type, $A_{V}$, and $L / L_{\mathrm{MS}}$ that we note in $\S 4.3 .2$ acts to preserve most of the inferred $V$-band excess under the assumption of a different spectral type.

One explanation that we cannot rule out is the presence of chromospheric activity or flaring. Stellar flares are characterized by transient regions with higher temperatures than the surrounding photosphere $\left(\gtrsim 10^{5} \mathrm{~K}\right)$; they cause a temporary increase in the total luminosity, with much higher amplitude of variability at short wavelengths. Surveys for X-ray emission from young BDs (e.g., Preibisch et al. 2005) have found that $1 / 3$ to $1 / 2$ of all objects may possess significant activity. These regions also typically produce $\mathrm{H} \alpha$ emission; the width of the base of the $\mathrm{H} \alpha$ emission line is a typical test for accretion, so it is possible that a large flare or significant chromospheric activity could broaden the line base, mimicking the signature of accretion.

\section{CONCLUSIONS}

We present the results of a high-resolution imaging survey of 22 brown dwarfs and very low mass stars in the nearby $(\sim 145 \mathrm{pc})$ young $(\sim 1-2 \mathrm{Myr})$ low-density star-forming region TaurusAuriga. This survey confirmed the binarity of MHO-Tau- 8 and discovered a new candidate binary system, V410-Xray3, resulting in a binary fraction of $9_{-3}^{+10} \%$ at separations $>4$ AU. Both binary systems are tight $(<10 \mathrm{AU})$, and they possess mass ratios of 0.75 and 0.46 , respectively. The binary frequency and separations are consistent with very low mass binary properties in the field, but the mass ratio of V410-Xray3 is among the lowest known. The binary frequency and separations are also consistent with the trends in multiplicity statistics observed for higher mass Taurus members, which suggest a gradual decline in both properties toward low masses; the implications are that the distinct binary statistics of low-mass systems are set in the formation process and that this formation process is similar to that which creates low-mass stars. These objects and a similar set in Upper Scorpius reveal another possible mass dependence of multiplicity; all five binaries and candidate binaries fall in the more massive half of the combined sample, implying either 
a decline in frequency or a shift to smaller separations for the lowest mass binaries. We also combine the survey detection limits with the models of Chabrier et al. (2000) to show that there are no planets or very low mass brown dwarfs with separations $>3 M_{\mathrm{J}}$ at projected separations $>40$ AU orbiting any of the Taurus members in our sample, implying that planetarymass companions at wide separations like $2 \mathrm{M} 1207 \mathrm{~b}$ are found with frequency $\lesssim 5 \%$. Finally, we observe significant optical excesses in the spectral energy distributions of most targets and conclude that the targets with spectroscopic signatures of accretion possess larger optical excesses than other young brown dwarfs and very low mass stars.
We thank the referee for a prompt, thorough, and helpful critique of this paper. This work is based on observations made with the NASA/ESA Hubble Space Telescope, obtained at the Space Telescope Science Institute, which is operated by the Association of Universities for Research in Astronomy, Inc., under NASA contract NAS 5-26555. These observations are associated with program 9853. This work also makes use of data products from the Two Micron All Sky Survey, which is a joint project of the University of Massachusetts and the Infrared Processing and Analysis Center, California Institute of Technology, funded by the National Aeronautics and Space Administration and the National Science Foundation.
Baraffe, I., Chabrier, G., Allard, F., \& Hauschildt, P. H. 1998, A\&A, 337, 403 2002, A\&A, 382, 563

Basri, G., \& Batalha, C. 1990, ApJ, 363, 654

Bate, M., \& Bonnell, I. 2005, MNRAS, 356, 1201

Bate, M., et al. 2003, MNRAS, 339, 577

Bessell, M., \& Brett, J. 1988, PASP, 100, 1134

Billeres, M., Billères, M., Delfosse, X., Beuzit, J.-L., Forveille, T., Marchal, L., \& Martín, E. L. 2005, A\&A, 440, L55

Bouy, H., et al. 2003, AJ, 126, 1526

Briceno, C., et al. 1998, AJ, 115, 2074 2002, ApJ, 580, 317

Burgasser, A., et al. 2003, ApJ, 586, 512

Calvet, N., et al. 2004, AJ, 128, 1294

Chabrier, G., Baraffe, I., Allard, F., \& Hauschildt, P. 2000, ApJ, 542, 464

Chauvin, G., et al. 2004, A\&A, 425, L29

Close, L. M., Siegler, N., Freed, M., \& Biller, B. 2003, ApJ, 587, 407

Close, L. M., et al. 2005, Nature, 433, 286

Cutri, R. M., et al. 2003, The IRSA 2MASS All-Sky Point Source Catalog (Pasadena: Caltech)

Delfosse, X., et al. 1997, A\&A, 327, L25

Delgado-Donate, E. J., Clarke, C. J., \& Bate, M. R. 2003, MNRAS, 342, 926

Duchenne, G. 1999, A\&A, 341, 547

Duquennoy, A., \& Mayor, M. 1991, A\&A, 248, 485

Fischer, D. A., \& Marcy, G. W. 1992, ApJ, 396, 178

Ghez, A., Neugebauer, G., \& Matthews, K. 1993, AJ, 106, 2005

Gizis, J. E., et al. 2000, MNRAS, 311, 385

Goodwin, S., \& Kroupa, P. 2005, A\&A, 439, 565

Gullbring, E., et al. et al. 1998, ApJ, 492, 323

Halbwachs, J., et al. 2003, A\&A, 397, 159

Hartigan, P., et al. 1991, ApJ, 382, 617

Hawley, S. L., et al. 2002, AJ, 123, 3409

Hillenbrand, L., \& White, R. J. 2004, ApJ, 604, 741

Hubber, D., \& Whitworth, A. 2005, A\&A, 437, 113

Kirkpatrick, J., et al. 1999, ApJ, 519, 802

Koerner, D., et al. 1999, ApJ, 526, L25

Kohler, R., Kunkel, M., Leinert, C., \& Zinnecker, H. 2000, A\&A, 356, 541

Kraus, A., et al. 2005, ApJ, 633, 452

Kroupa, P. 1995, MNRAS, 277, 1522

Kroupa, P., \& Bouvier 2003, MNRAS, 346, 343

Kroupa, P., et al. 2003, MNRAS, 346, 354

\section{REFERENCES}

Leggett, S., et al. 2000, ApJ, 536, L35 2002, ApJ, 564, 452

Leinert, C, et al. 1993, A\&A, 278, 129

Luhman, K. L. 2004a, ApJ, 614, 398 2004b, ApJ, 617, 1216

2005, ApJ, 633, L41

Luhman, K. L., Briceño, C., Stauffer, J. R., Hartmann, L., Barrado y Navascués, D., \& Caldwell, N. 2003a, ApJ, 590, 348

Luhman, K. L., McLeod, K. K., \& Goldenson, N. 2005a, ApJ, 623, 1141

Luhman, K. L., Stauffer, J. R., \& Mamajek, E. E. 2005b, ApJ, 628, L69

Luhman, K. L., Stauffer, J. R., Muench, A. A., Rieke, G. H., Lada, E. A., Bouvier, J., \& Lada, C. J. 2003b, ApJ, 593, 1093

Mack, J., et al. 2003, ACS Data Handbook, Version 2.0 (Baltimore: STScI)

Martin, E. L., Dougados, C., Magnier, E., Ménard, F., Magazzù, A., Cuillandre,

J.-C., \& Delfosse, X. 2001, ApJ, 561, L195 2003, ApJ, 594, 525

Metchev, S. A., Hillenbrand, L. A., \& White, R. J. 2003, ApJ, 582, 1102

Mohanty, S., Jayawardhana, R., \& Basri, G. 2005, ApJ, 626, 498

Muzerolle, J., et al. 2003, ApJ, 592, 266

. 2005, ApJ, 625, 906

Neuhauser, R., et al. 2002, A\&A, 384, 999

Phan-Bao, N., Martín, E. L., Reylé, C., Forveille, T., \& Lim, J. 2005, A\&A, 439, L19

Preibisch, T., et al. 2005, ApJS, 160, 401

Reid, I. N., \& Gizis, J. E. 1997, AJ, 113, 2246

Reid, I. N., \& Hawley, S. L. 1999, AJ, 117, 343

Reid, I. N., et al. 2004, AJ, 128, 463

Reiners, A., Basri, G., \& Mohanty, S. 2005, ApJ, 634, 1346

Reipurth, B., \& Clarke, C. 2001, AJ, 122, 432

Schlegel, D., Finkbeiner, D., \& Davis, M. 1998, ApJ, 500, 525

Siegler, N., et al. 2005, ApJ, 621, 1023

Simon, M., et al. 1995, ApJ, 443, 625

Stassun, K., Mathieu, R. D., \& Valenti, J. A. 2006, Nature, 440, 311

Sterzik, M., \& Durisen, R. 1998, A\&A, 339, 95

Stetson, P. B. 1987, PASP, 99, 191

Strom, K. M., \& Strom, S. E. 1994, ApJ, 424, 237

West, A. A., Walkowicz, L. M., \& Hawley, S. L. 2005, PASP, 117, 706

White, R. J., \& Basri, G. 2003, ApJ, 582, 1109

White, R. J., \& Ghez A. M. 2001, ApJ, 556, 265

White, R. J., \& Hillenbrand, L. A. 2004, ApJ, 616, 998 